\title{
Erosional Furrows Formed During the Lateral Blast at Mount St. Helens, May 18, 1980
}

\author{
SUSAN WERNER KIEFFER \\ U.S. Geological Survey, Flagstaff, Arizona \\ BRADFORD STURTEVANT \\ California Institute of Technology, Pasadena
}

\begin{abstract}
Nearly horizontal, quasi-periodic erosional features of 7-m average transverse wavelength and of order 100-m length occur in scattered locations from 3.5 to $9 \mathrm{~km}$ from the crater at Mount St. Helens under deposits of the lateral blast of May 18, 1980. We attribute the erosional features to scouring by longitudinal vortices resulting from flow instabilities induced by complex topography, namely, by streamline curvature in regions of reattachment downstream of sheltered regions, and by the cross-flow component of flow subparallel to ridge crests. The diameter of the vortices and their transverse spacing, inferred from the distance between furrows, are taken to be of the order of the boundary layer thickness. The inferred boundary layer thickness $(\approx 14 \mathrm{~m}$ at $9 \mathrm{~km}$ from the source of the blast $)$ is consistent with the running length from the mountain to the furrow locations. By using knowledge of ablation patterns on bodies and lofting of dust in high-speed flow, we are able to infer some features of the flow field within the blast. Within the furrows the erosion rate was of the order of $9 \mathrm{~kg} \mathrm{~m}^{-2} \mathrm{~s}^{-1}$, about 4 times greater than that expected from laboratory data obtained in flow free of longitudinal vortices. The orientation of furrows induced by the cross-flow instability can be used to measure the upwash angle and estimate the flow Mach number: at the central ridge of Spirit Lake the Mach number is inferred to have been about 2.5, and the flow velocily approximately $235 \mathrm{~m} / \mathrm{s}$. The similarities and differences between the furrows reported here and channels observed at other volcanoes are discussed.
\end{abstract}

\section{INTRODUCTION}

A volcano can be viewed as a source from which occasional disruptions in the sedimentary sequence of adjacent terrain originate. At the volcano an eruption has one or more waxing and waning stages. Historically, reconstruction of the dynamics of explosive volcanic eruptions has relied mainly on studies of deposits left during the waning phases of flows, blasts, surges, or Plinian columns. However, as pointed out by Fisher [1977], keys to the understanding of eruption dynamics also may be imprinted on a landscape during the waxing stages of eruptions, particularly when pyroclastic flows and surges are involved. Few observations have been made relevant to the erosional waxing stage because it is manifested as an eroded surface, not a new deposit. Such surfaces are difficult to recognize or interpret in cross section and are rarely revealed in three dimensions. Yet much of the destructive power of volcanic eruptions is exerted during the formation of these surfaces, and their features may carry important information about the early development of blasts, surges, and pyroclastic flows. Understanding the processes by which such erosion surfaces are created is of fundamental importance to volcanic hazard studies.

There is a rare opportunity to study eruption-caused erosion at Mount St. Helens because expressions of the erosional stage, including sand-blasted trees and scoured landscape, are ubiquitous and are well exposed or only thinly covered by blast deposits. We describe erosional leatures, which we call furrows, created by the lateral blast of May 18, 1980, at Mount St. Helens. The furrows are similar in cross section and

\section{Copyright 1988 by the American Geophysical Union.}

Paper number 7 B 7170 .

0148-0227/88/007B-7170\$05.00 dimension to radial troughs observed on the cones of Taal Volcano [Moore, 1967], Bárcena Volcano, Mexico [Richards, 1959; Fisher, 1977], Cerro Colorado, Mexico [Wohletz, 1977], Latera and Baccano craters, Italy [Losacco and Parea, 1969; Mattson and Alvarez, 1973], and Beagle Crater, Galapagos [Darwin, 1896], but may differ in depositional and/or erosional relations to underlying and overlying deposits. They seem to be different from the larger channels described by Rowley et al. [1981, p. 509] cut by pyroclastic flows on the flanks of Mount St. Helens, and the U-shaped channels reported by Fisher [1977] at Koko Crater, Hawaii, and Fisher and Schminke [1984, p. 257] at Lacher See, Germany, which may have been cut by the lobes of base surges. The furrows at Mount St. Helens are distinguished by the distance from the source at which they occur and by subhorizontal orientations, in contrast to downslope orientations at most of these other locations.

The furrows always occur in groups consisting of tens of individual furrows, and they typically occur on or near ridges. In this respect they are similar in form and distribution to familiar ablation patterns which form on nose cones of missiles and tektites during entry into the Earth's atmosphere. That is, during the lateral blast at Mount St. Helens the ridges were analogous to bodies in high-speed atmospheric flight or in high-speed wind tunnels. This analogy provides the opportunity to use knowledge of ablation and lofting of dust in high-speed flow to characterize the blast flow at Mount St. Helens. In particular, we attribute the furrows to erosion by longitudinal vortices, that is, vortices aligned with the direction of flow. In order for such prominent and regular erosional features to occur, there must be strong interaction between the vortex flow and the eroding geometry of the ground, just as with the formation of ablation patterns; presumably, the formation of furrows enhances the strength and coherence of the 
TABLE 1. Locations of Furrows at Mount St. Helens

\begin{tabular}{|c|c|c|c|}
\hline Locality & $\begin{array}{l}\text { Coordinates } \\
\text { (Min From } \\
46^{\circ} \mathrm{N} 122^{\circ} \mathrm{W} \text { ) }\end{array}$ & $\begin{array}{l}\text { Section of } \\
\text { T9N R5E }\end{array}$ & Remarks \\
\hline \multicolumn{4}{|c|}{ Studebaker Ridge } \\
\hline S1 & $13.6^{\prime} \mathrm{N} 13.4^{\prime} \mathrm{W}$ & SE Qtr Sec 30 & Preblast forestation unknown \\
\hline S1a & $13.5^{\prime} \mathrm{N} 13.4^{\prime} \mathrm{W}$ & NE Qtr Sec 31 & \\
\hline Slb & $13.6^{\prime} \mathrm{N} 13.3^{\prime} \mathrm{W}$ & SE Qtr Sec 31 & \\
\hline S2 & $13.5^{\prime} \mathrm{N} 13.2^{\prime} \mathrm{W}$ & NE Qtr Sec 31 & $\begin{array}{l}\text { Sites at localities S2-S11 (N and S) identified } \\
\text { on air photos but not verified in the field }\end{array}$ \\
\hline S3 & $13.5^{\prime} \mathrm{N} 13.1^{\prime} \mathrm{W}$ & NE Qtr Sec 31 & \\
\hline S4 & $13.4^{\prime} \mathrm{N} 13.1^{\prime} \mathrm{W}$ & NW Q1r Sec 32 & \\
\hline \$5 & $13.2^{\prime} \mathrm{N} 13.1^{\prime} \mathrm{W}$ & NE Qtr Sec 31 & \\
\hline S6 & $13.9^{\prime} \mathrm{N} 13.4^{\prime} \mathrm{W}$ & SE Qtr Sec 30 & \\
\hline S7 & $13.7^{\prime} \mathrm{N} 13.5^{\prime} \mathrm{W}$ & SE Qtr Sec 30 & \\
\hline S8 & $13.9^{\prime} \mathrm{N} 13.8^{\prime} \mathrm{W}$ & SW Qtr Sec 30 & \\
\hline S9 & $13.0^{\prime} \mathrm{N} 14.0^{\prime} \mathrm{W}$ & SW Qtr Sec 30 & \\
\hline S10 & $13.7^{\prime} \mathrm{N} 13.2^{\prime} \mathrm{W}$ & SE Qtr Sec 30 & \\
\hline S11N & $14.7^{\prime} \mathrm{N} 13.5^{\prime} \mathrm{W}$ & SE Qtr Sec 19 & \\
\hline S11S & $14.5^{\prime} \mathrm{N} 13.4^{\prime} \mathrm{W}$ & SE Qtr Sec 19 & \\
\hline \multirow{2}{*}{\multicolumn{4}{|c|}{$\begin{array}{l}\text { Windy Ridge } \\
\text { and Smith Creek }\end{array}$}} \\
\hline & & & \\
\hline W1 & $14.2^{\prime} \mathrm{N} 9.0^{\prime} \mathrm{W}$ & NW Qtr Sec 26 & \\
\hline W2 & $14.4^{\prime} \mathrm{N} 8.7^{\prime} \mathrm{W}$ & NE Qtr Sec 26 & \\
\hline W3 & $14.3^{\prime} \mathrm{N} 8.6^{\prime} \mathrm{W}$ & NE Qtr Sec 26 & \\
\hline W4 & $14.4^{\prime} \mathrm{N} 8.5^{\prime} \mathrm{W}$ & NE Qtr Sec 26 & $\begin{array}{l}\text { Sites at localities W4-W6 identified on air } \\
\text { photos but not verified in the field }\end{array}$ \\
\hline W5 & $14.4^{\prime} \mathrm{N} 8.4^{\prime} \mathrm{W}$ & NE Qtr Sec 26 & \\
\hline W6 & $14.3^{\prime} \mathrm{N} 8.7^{\prime} \mathrm{W}$ & NE Qtr Sec 26 & \\
\hline \multicolumn{4}{|l|}{ Johnston Ridge } \\
\hline J1 & $16.9^{\prime} \mathrm{N} 12.7^{\prime} \mathrm{W}$ & NW Qtr Sec 8 & Virgin forest, both sides FR4024 \\
\hline $\mathrm{J} 2$ & $17.0^{\prime} \mathrm{N} 12.9^{\prime} \mathrm{W}$ & NW Qtr Sec 8 & Steep slope, edge of forest \\
\hline $\mathrm{J} 2 \mathrm{a}$ & $17.0^{\prime} \mathrm{N} 13.0^{\prime} \mathrm{W}$ & NW Qtr Sec 8 & Clear-cut, in drainage \\
\hline $\mathrm{J} 2 \mathrm{~b}$ & $17.0^{\prime} \mathrm{N} 13.2^{\prime} \mathrm{W}$ & SE Qtr Sec 6 & Virgin forest, ridge slope \\
\hline J3 & $16.9^{\prime} \mathrm{N} 13.4^{\prime} \mathrm{W}$ & NE Qtr Sec 7 & Clear-cut, Ohanapecosh Formation outcrop \\
\hline $\mathrm{J} 3 \mathrm{a}$ & $16.7^{\prime} \mathrm{N} \mathrm{13.3^{ \prime } \mathrm { W }}$ & NE Qir Sec 7 & Clear-cut, in drainage \\
\hline $\mathrm{J} 4$ & $17.0^{\prime} \mathrm{N} 13.9^{\prime} \mathrm{W}$ & NW Qtr Sec 7 & Virgin forest, site unverified \\
\hline $\mathrm{J} 5$ & $16.8^{\prime} \mathrm{N} 13.2^{\prime} \mathrm{W}$ & NE Qtr Sec 7 & Clear-cut, site unverified \\
\hline \multicolumn{4}{|l|}{ Harry's Ridge } \\
\hline HI & $16.8^{\prime} \mathrm{N} 10.3^{\prime} \mathrm{W}$ & NW Qtr Sec 10 & Virgin forest, extensive area west of crest \\
\hline $\mathrm{H} 2$ & $16.8^{\prime} \mathrm{N} 10.2^{\prime} \mathrm{W}$ & NW Qtr Sec 10 & Virgin forest, extensive area east of crest \\
\hline \multicolumn{4}{|l|}{ Central Ridge } \\
\hline $\mathrm{Cl}$ & $16.7^{\prime} \mathrm{N} 8.7^{\prime} \mathrm{W}$ & NW Qtr Sec 1I & Adjacent to Spirit Lake run-up trim line \\
\hline
\end{tabular}

vortices, while the increased vortical intensity hastens erosion. Coherent vorticity in turbulent flow, e.g., large eddies in the atmosphere and ocean, plays an important role in fluid mixing and transport [Roshko, 1976]. In this paper we document this example of enhanced erosion by large-scale vortices in a volcanic setting, and we estimate the Reynolds number, Mach number, Görtler number, and erosional efficiency of the blast at Mount St. Helens.

\section{DESCRIPTION}

The furrows at Mount St. Helens are exposed in a zone between the area in which most trees (and much of the thin forest litter) have been removed by the blast and/or buried by the debris avalanche, and the distal region where trees are downed but appear nearly in place. As listed in detail in Table 1 and summarized in Figure 1, some of the furrows are found in a proximal zone $3.5-5.5 \mathrm{~km}$ from the origin of the blast in two areas outside of the sector scoured and overlain by the debris avalanche. These two areas bear $50^{\circ}$ west and east, respectively, of the central axis along which the blast was directed. The proximal furrows are best exhibited at Studebaker Ridge ( $S$ in Figure 1; Figure 2) and Windy Ridge (W in Figure 1). Furrows also occur in a distal arc 8-9 km from the source, bearing from $20^{\circ}$ west to $20^{\circ}$ east of north. In this region they are especially prominent at Johnston Ridge ( $\mathrm{J}$ in Figure 1; Figure 3), Harry's Ridge ( $\mathrm{H}$ in Figure 1; Figures 4 and 5), and the unnamed central ridge extending south into Spirit Lake (C in Figure 1; Figure 6). The furrows at Johnston Ridge occur on the north (lee) side of the ridge as far as $290 \mathrm{~m}$ in altitude below the crest.

The furrows lie in specific zones of tree abrasion, blast deposit thickness, and average largest particle size in the blast deposit (J. G. Moore, oral communication, 1987). As shown by the contours in Figure $1 a$, abrasion on small trees that were salvaged from these zones was typically $50-100 \mathrm{~mm}$. Figure $1 b$ shows that the furrowed areas tend to fall where the thickness of the coarse basal unit of Moore and Sisson [1981] exhibits large variations between 0 and $100 \mathrm{~mm}$. Though the variations observed in our work of the thickness of this deposit from one furrowed area to another are consistent with the trends indicated in Figure $1 b$ (cf. Table 2), our observations did not disclose significant variations of deposit thickness within any given furrowed area. Figure $1 c$ shows that the largest clasts found in the basal unit were typically $10 \mathrm{~cm}$ or larger.

Within the zone where furrows occur, the entire landscape 

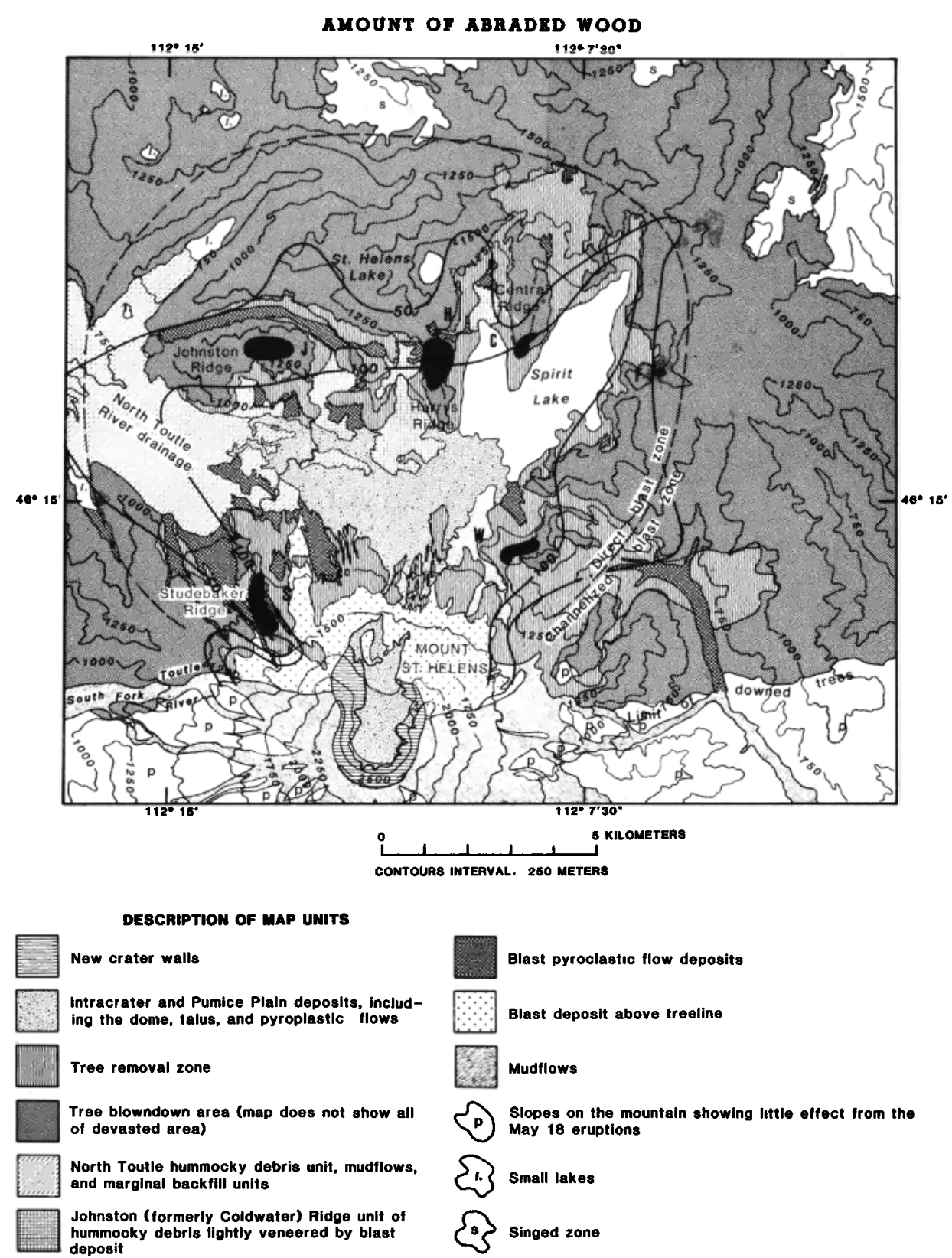

Fig. $1 a$

Fig. 1. Locations of furrows documented to date, superimposed on a simplified topographic and geologic map of Mount St. Helens showing the deposits associated with the 1980 and subsequent eruptions. The furrowed areas that have been identified from air photos and/or verified in the field are shown in black; the Studebaker Ridge (S) and Windy Ridge (W) localities are in the "proximal zone," and the Johnston Ridge (J), Harry's Ridge (H), central ridge extending into Spirit Lake (C), and Harmony Falls (F) localities are in the "distal zone." See Table 1 for specific locations. The dashed line separating the direct and channelized blast zones indicates the approximate boundary between an inner area where the direction of tree fall and inferred blast direction is relatively independent of large-scale topography and an outer zone where the direction of tree fall and inferred blast direction depends quite strongly on relatively subtle topography (see Kieffer [1981] for details). (a) Superposed contours showing thickness of wood removed from the sides of small trees and stumps facing the volcano (in millimeters.) Note that most furrowed areas lie close to the 100 -mm contour, and all are nearly confined between the 50- and 100-mm contours. All contours in Figures 1a-1c are from Moore and Sisson [1981]; see that reference for details. (b) Superposed contours of average thickness of coarse basal unit of blast (in centimeters). This unit consists of layers A0 and A1 of Fisher et al. [1987]. Although it is not obvious from this map because flow directions are not explicitly shown, the contours show that the furrowed zones occur where the thickness of the basal blast deposit decreases dramatically in the downstream direction (with the exception of the Johnston Ridge location). (c) Average diameter of the three largest clasts within the surge deposit (in centimeters). With the exception of the Harmony Falls area, the largest clasts tend to exceed $10 \mathrm{~cm}$ in the furrowed areas. The base map in Figures $1 a-1 c$ is simplified from a geologic map from Lipman and Mullineaux [1981]. 


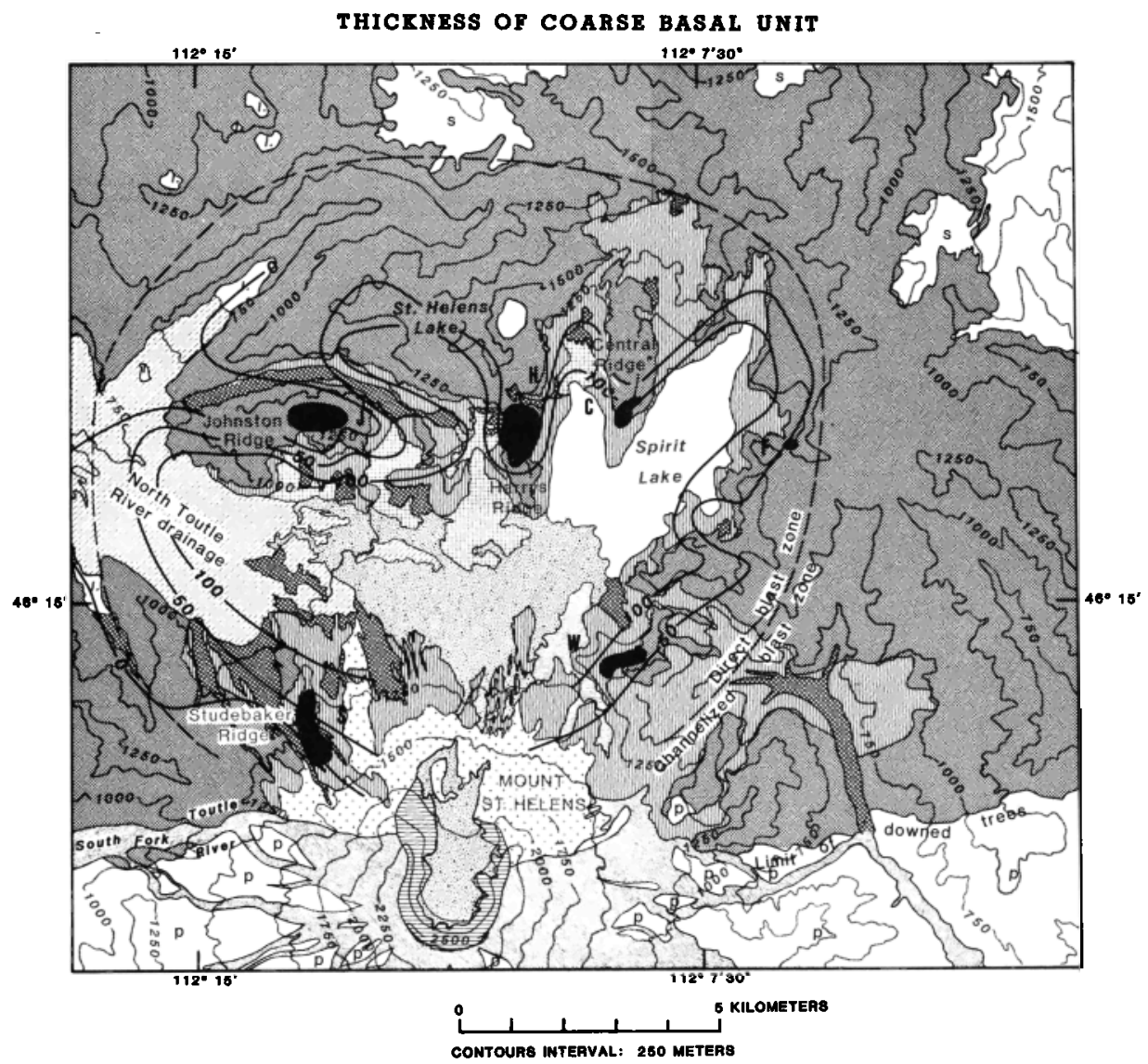

DESCRIPTION OF MAP UNITS

New erater walls

Intracrater and Pumice Plain deposits, includIng the dome, talus, and pyroplastic tlows

Tree removal zone

Tree blowndown area (map does not show all of devasted area)

North Toutle hummocky debris unil, mudflows. and marginal back fill units

Johnston (formerly Coldwater) Ridge unit of hummocky debris lightly veneered by blast deposil
Blast pyroclastic flow deposits

Blast deposit above treeline

Mudflows

Slopes on the mountain showing little effect from the May 18 eruptions

S. small lakes

$\int 3$ singed zone

Fig. $1 b$ was lowered to tree-root level (for Douglas fir trees, the root level is typically $0.3-0.5 \mathrm{~m}$ below the litter surface (K. Graves, oral communication, 1987)), and additional erosion occurred within the furrows. Between furrows therefore root systems and some of their host soils/deposits remain, whereas this material has been removed within the furrows. Partial filling of the furrows by deposits from the lateral blast subdues the erosional corrugated profiles and gives furrowed hillsides a terraced appearance. The axes of the furrows are nearly parallel to the local direction of the blast (as inferred from abrasion on tree trunks and the orientation of downed trees) and are usually within about $10^{\circ}$ of horizontal. In the field, relationships between furrows, grooves, the avalanche trim line, and water runoff channels need to be carefully distinguished (e.g., as on Harry's Ridge where Figure 4 shows the avalanche trim line, a landslide scarp, and the furrows).

Although the locations where furrows have been found are limited (it may be that some locations where they would have been found are concealed by the landslides and pyroclastic flows north and east of the mountain), where furrows have been found, they occur in large numbers. For example, they cover the headwall at South Coldwater Creek on Harry's Ridge, and on the east side of Harry's Ridge they occur in a band extending $220 \mathrm{~m}$ in altitude below the summit (Figure 4). Although now prominent, the furrows went unnoticed for several years after the May 18, 1980, eruption because the local detailed topography caused by the furrows was mantled by the postblast ash deposits. By 1985, recognition of the furrows from the air had been enhanced by partial erosion of the ash deposits which wash downslope into low points in the furrows. Whereas in the early 1980 s the light color of the ash deposits covering the furrows tended to make recognition of them difficult, now that the ash deposits lie preferentially within the low points of the furrows, the light color of the ash actually highlights the presence of the furrows (Figure 7). Erosion is, however, degrading the furrows: rainwater ponds in them, flows 

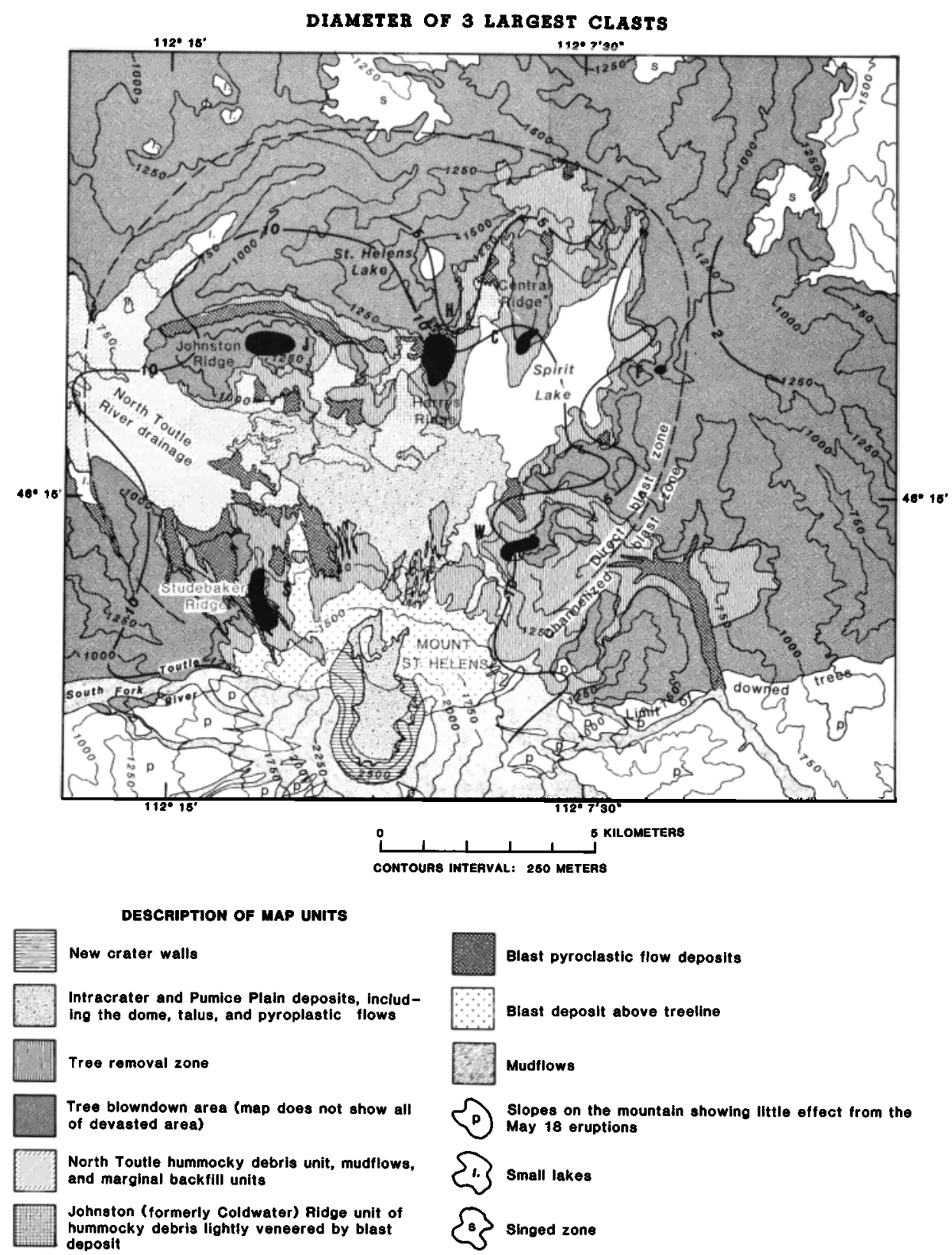

Fig. 1c

along them longitudinally where their axes are gently dipping, and then typically breaks out to form narrow gullies that run downslope. Thus in air photos, furrowed slopes often exhibit a nearly orthogonal mesh of furrows and gullies (Figure $7 b$ ). Figures 8 and 9 show the influence of furrows on the development of gullies in more detail. The subdivision of the long furrows into short sections by the gullying process (perhaps within days to months after furrow formation) probably contributes to the problem mentioned above of recognizing furrows in the stratigraphic record.

Where deposits from ash falls subsequent to the morning of May 18, 1980, were heavy, as in the upper Smith Creek drainage, the furrows are still nearly buried and often almost indistinguishable. At such localities it has not been practicable to dig through the thick deposits to study them. Table 1 lists the localities at which furrows have been found during field surveys or identified on aerial photographs. There seems to be no correlation between the propensity for furrows to form and the state of preblast forestation (e.g., virgin forest versus clearcut land).

Furrows occur in two settings distinguished by different relations between topography and fluid-mechanical environments, illustrated in Figures 1 and 4. (Laboratory experiments with experimental analogs of these settings are discussed in the last part of this paper and are shown in Figure 15.) In the first setting, furrows are found downstream (relative to the local direction of the lateral blast) of patches of remnant trees and stumps that were preserved in topographically sheltered places. This geometry is illustrated in both map view and cross section in Figure 4, which shows how the blast flowed up the prow of Harry's Ridge, detached when it reached the sudden change in curvature at the summit, and reattached about $240 \mathrm{~m}$ downstream on the west slope. The west slope of Harry's Ridge is a concave cirque; a possible effect of this curvature on the flow is discussed in section 4 . In the sheltered region downstream of the detachment point there was a rela- 


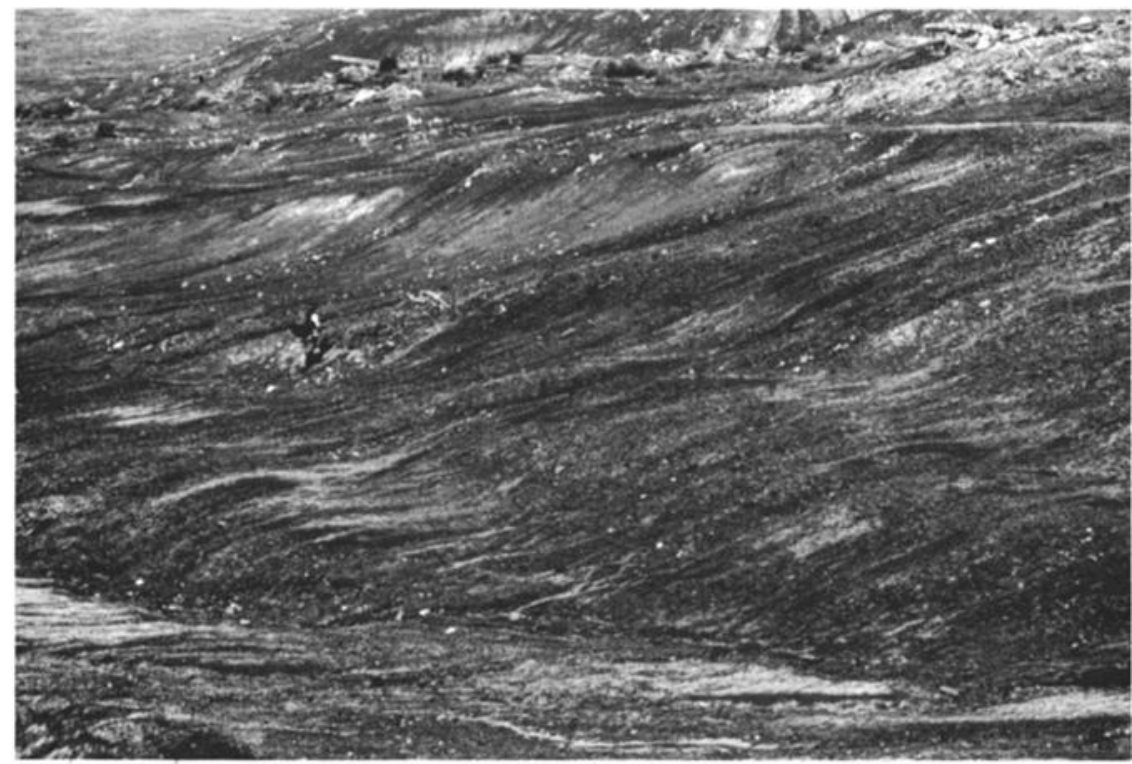

Fig. 2. Furrows of about 5-m wavelength at locality S1, Studebaker Ridge. Looking north toward North Fork Toutle River. The primary mechanism of erosion at Studebaker Ridge appears to have been furrowing by cross-flow instability (see section 3 for discussion).

tively low-energy recirculating motion. Evidence of the recirculation can be seen in the conical shapes of the remnant tree stumps in such regions: the stumps were eroded on the windward side by direct impact of the blast, and on the leeward side by abrasion by the recirculating flow (Figure 10). Although there are remnant stumps and, in some cases, trunks in the sheltered zones upstream of the furrows (Figure $4 b$ ), the furrowed slopes downstream of reattachment are highly eroded and conspicuously free of tree trunks.

In the second setting, furrows occur on and near the crests of ridges oriented subparallel to the inferred flow direction (Figure $1 b$, the central ridge and Studebaker Ridge; Figures 2 and 6). In such circumstances the component of flow across the ridge (cross flow) could lead to furrow formation (see section 3). The furrows on the central ridge extending into Spirit Lake trace a helical pattern from one side of the ridge across the crest to the other. The most prominent example of such a helical furrow is shown in Figure 11.

Prominent furrows also occur near the prow of the central ridge on terrain that was washed by the avalanche-generated wave in Spirit Lake. They are very regular and somewaht larger in scale than the ones above the wave run-up line, and they may exhibit crosshatching (see section 3). It is not known whether they were generated before, during, or after the wave run-up. Because the circumstances of their formation may be more complex, we do not consider them further in this work.

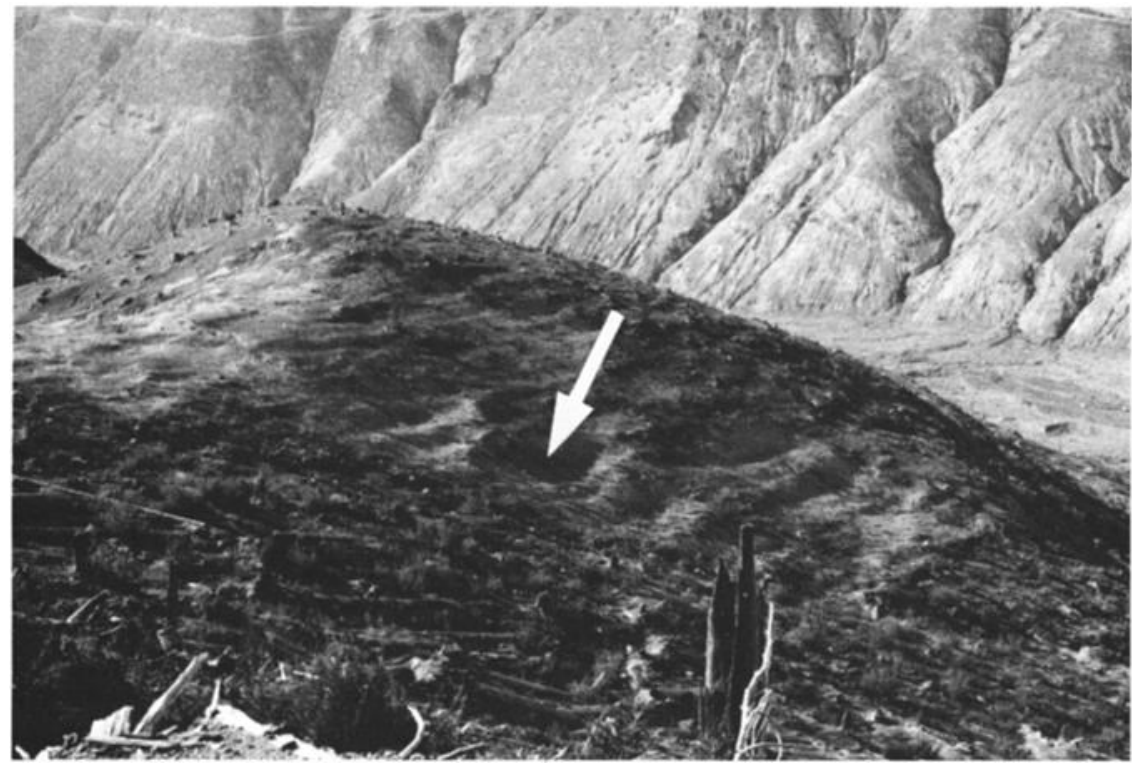

Fig. 3. Furrows on north side of Johnston Ridge (locality J2) overlooking South Coldwater Creek. Widest furrow (J2-I5, shown by arrow) is $16 \mathrm{~m}$ wide from crest to crest. Furrows at this site occur between a sheltered separated-flow region in the foreground and a convex lightly eroded crest just downstream, and are notable for their short length (see section 4 for discussion). 


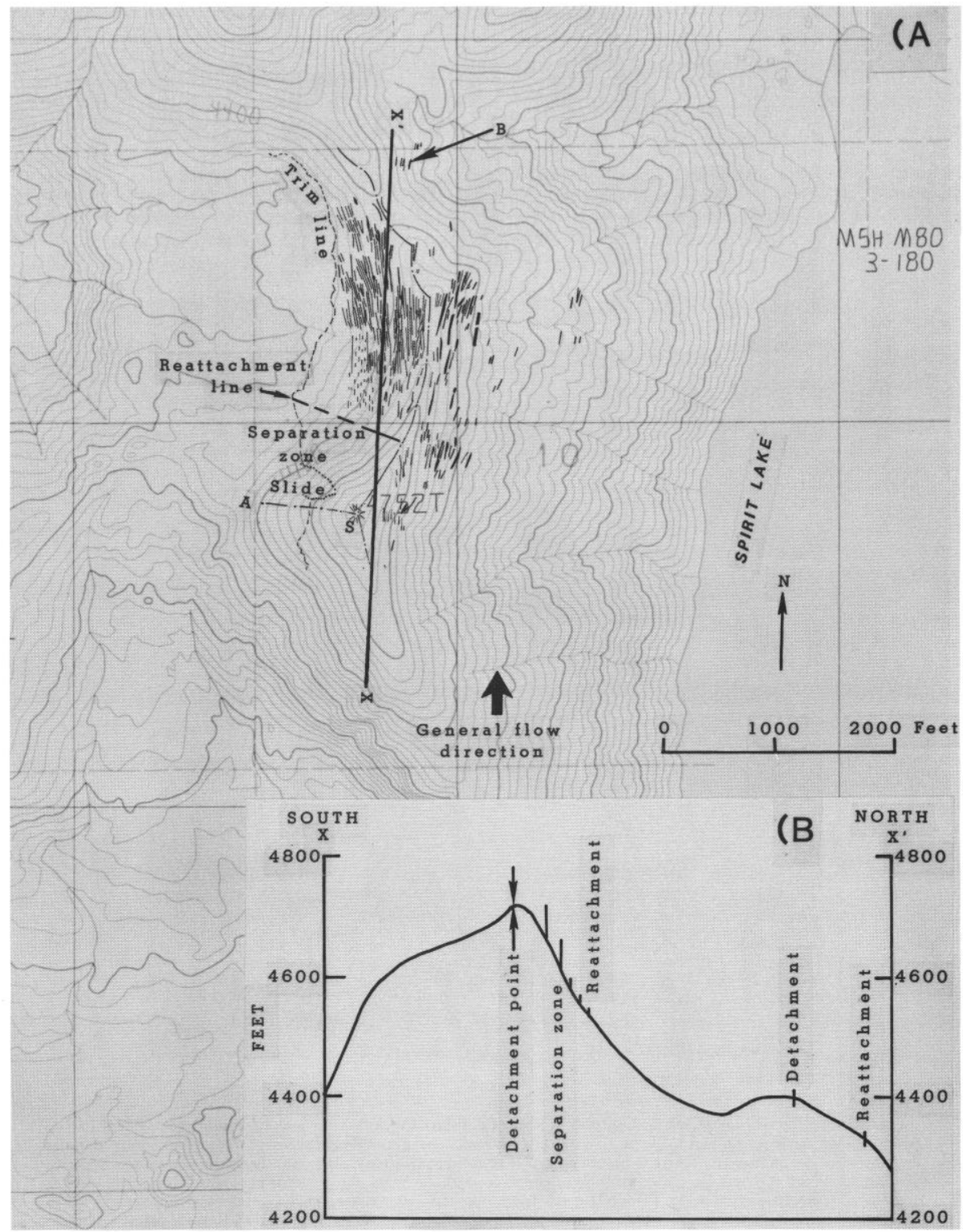

Fig. 4. (a) Map of Harry's Ridge showing the distribution of individual furrows (solid lines) identified on aerial photos 25-3 and 26-11 of black-and-white series of the USGS dated July 7, 1984. Many of these furrows were verified in the field. Others, observable in the field or on other air photos, are shown as short dashes. The continuous dashed line is the trim line of the debris avalanche; dotted line is scarp of slump of soil and rock; dash-dot lines are ridge crests. Average flow direction shown by heavy arrow. Separation of flow occurred approximately along crest A-S, and reattachment occurred upstream of the furrows along the indicated reattachment line (heavy dashed where estimated). Note second small zone of separation and reattachment at the saddle near area B. (b) Cross section along X-X', showing separation and reattachment lines. The vertical lines in the separation zone represent the decreasing height of sheared trees from ridge crest to the reattachment line. From such data, we estimate a radius of curvature for the reattaching flow to be of the order of $100 \mathrm{~m}$. 


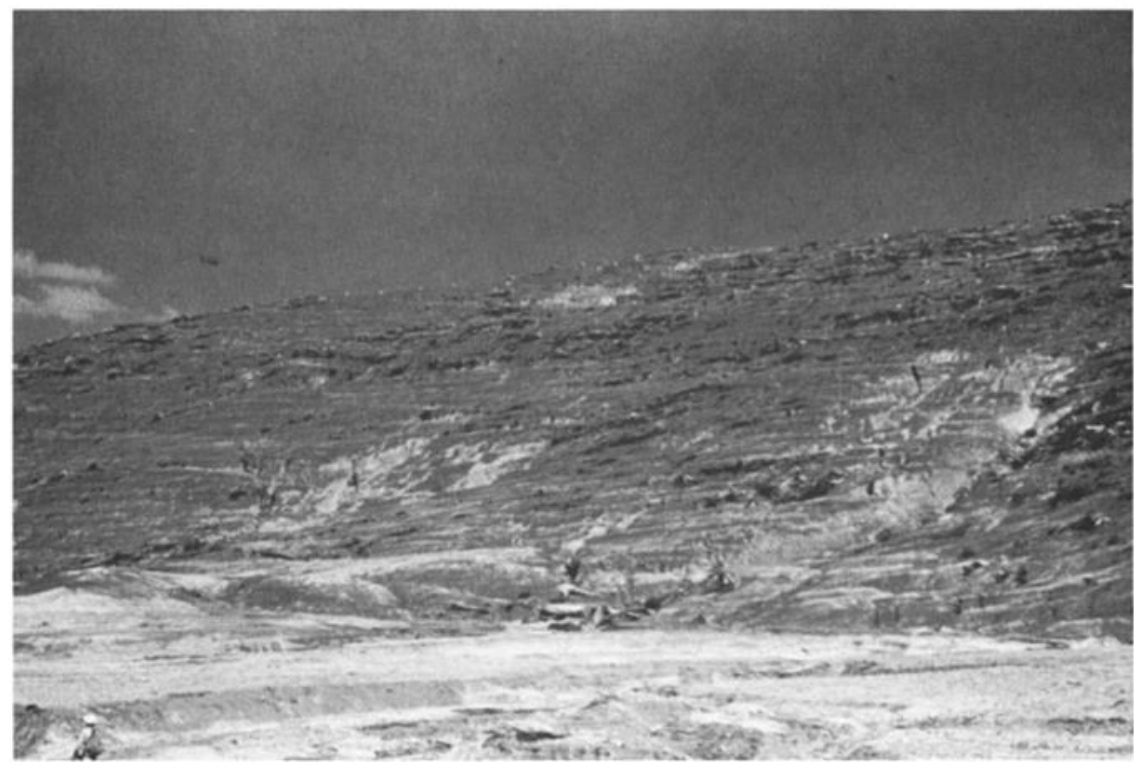

Fig. $5 a$

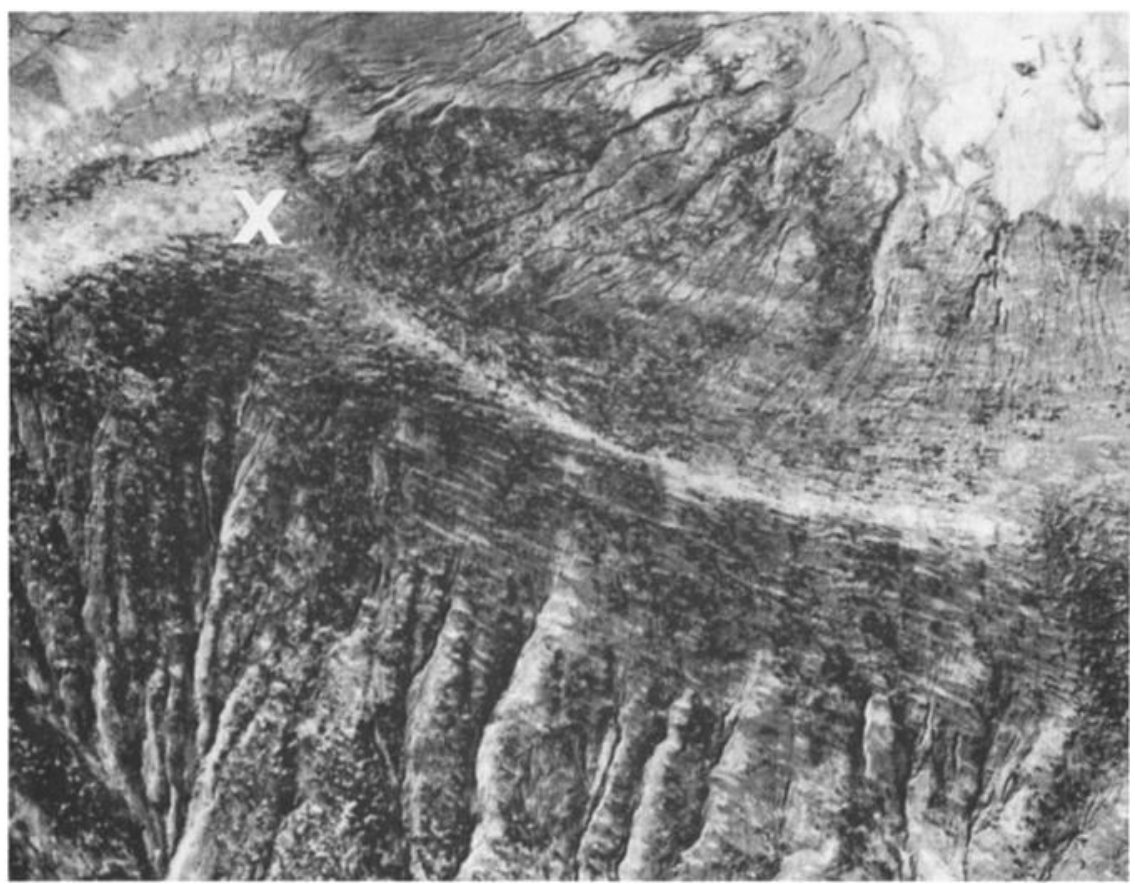

Fig. $5 h$

Fig. 5. (a) Furrowed hillside at the headwall of South Coldwater Creek. View is looking east at the northern part of Harry's Ridge. Several tens of furrows give the slope a terraced appearance. Avalanche deposits cover the foreground at the bottom of the photograph. There is no evidence that the avalanche rode higher onto the hillside than these deposits. Distance along the skyline ridge shown in the photograph is $600 \mathrm{~m}$. (b) High-altitude aerial photograph (from USDA-F 12 616030b 1285-B4, dated August 25, 1985) of Harry's Ridge, looking west, showing furrows on its eastern flank. At top of photograph is the headwall of South Coldwater Creek, shown in Figure 5a. Summit of Harry's Ridge is marked by cross at left top. View shows extensive furrowing from south of the summit to the sheltered region at extreme right. Distance along the ridge from the summit to the bifurcation point at extreme right center is $600 \mathrm{~m}$.

In general, furrows are partly filled with deposits of the lateral blast, but often the deepest furrows are almost completely free of blast deposits (e.g., at locality J2, Figure 3). The fact that the slopes immediately upstream (e.g., in the near foreground, Figure 3) and downstream of the furrowed slopes in the figure are mantled with blast deposits suggests that during the depositional phase enough flow energy remained to maintain the furrowing mechanism. In locations where the furrows are partly filled with deposits, by the time deposition began the furrows had already been formed, so they were rapidly filled by mobile blast debris. In these cases the true eroded surface can only be recognized in erosional gullies that 


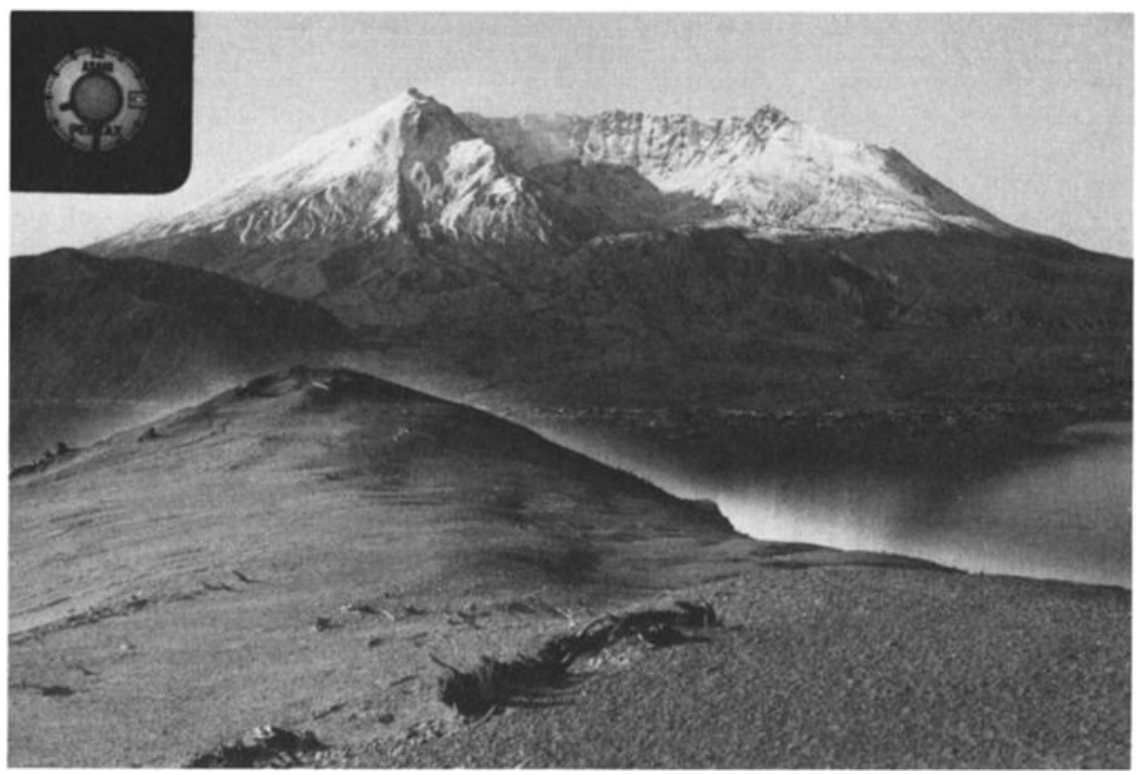

Fig. $6 a$

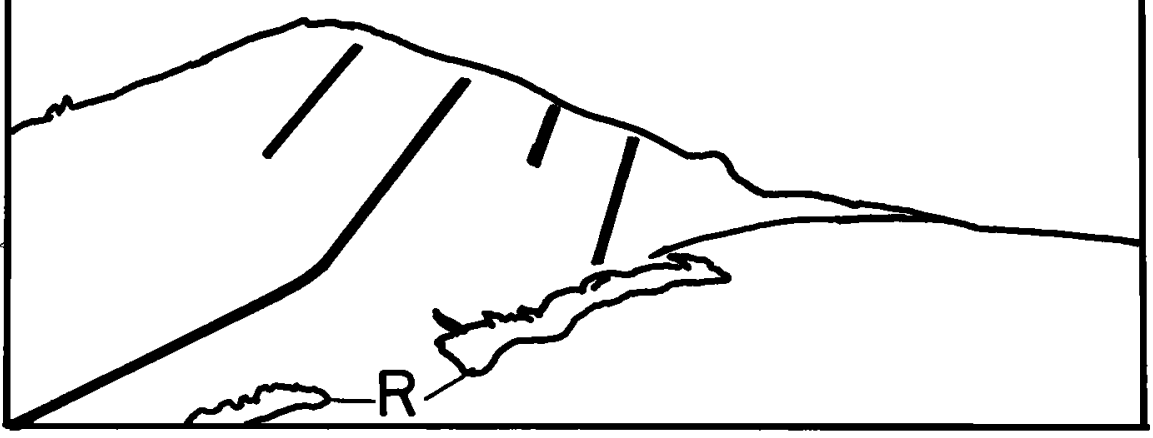

Fig. $6 b$

Fig. 6. (a) View of furrows on the crest of the central ridge (locality C1) looking south. Furrows originate at the right ridgeline in the center of this view and extend to the left toward the camera at an average angle of $6^{\circ}$ greater than the bearing to the center of the crater. Distance from furthest point on ridge crest to photographer is $150 \mathrm{~m}$. (b) Schematic illustration of the centerlines (heavy solid lines) of the furrows of Figure $6 a$.

transect the furrows or in trenches (Figure 12). Deposition into the furrows during a post-erosional phase may have occurred by two processes, one in which the furrows acted as receptacles for direct deposit of waning phase material, and the other in which they acted as receptacles for the downslope movement of mobile blast ejecta.

Remnants of timber removed from furrowed slopes cannot be found downstream of the furrows (for example, in the next sheltered region downstream, as in the second sheltered zone on Harry's Ridge shown in Figure 4b). We infer therefore that the trees and bushes on furrowed slopes were shredded to small pieces almost immediately after they were reached by the blast and carried away. One exception occurs just downstream of the reattachment line at locality $\mathrm{H} 1$ on Harry's Ridge, where organic-rich basal blast deposit (designated AO by Fisher et al. [1987]) has been found in the bottom of furrows under A1 blast deposit. We interpret its presence in the furrows near the reattachment zone but not further downstream to mean either that tree shedding in the large sheltered region at Harry's Ridge continued into the waning stages of the blast or that large amounts of organic and blast debris were trapped for the duration of the blast in the recirculating flow there.

As discussed in section 3, it is likely that local features of the 
TABLE 2. Furrow Dimensions

\begin{tabular}{|c|c|c|c|c|c|c|c|}
\hline \multirow[b]{2}{*}{ Furrow } & \multirow[b]{2}{*}{ Area, $\mathrm{m}^{2}$} & \multirow{2}{*}{$\begin{array}{c}\text { Slope } \\
\text { Distance, m }\end{array}$} & \multirow{2}{*}{$\begin{array}{c}\text { Slope } \\
\text { Width, } m\end{array}$} & \multicolumn{2}{|c|}{ Depth, ${ }^{*} \mathrm{~cm}$} & \multirow{2}{*}{$\begin{array}{l}\text { Crest-to-Crest } \\
\text { Slope, deg }\end{array}$} & \multirow{2}{*}{$\begin{array}{c}\text { Blast } \\
\text { Deposit } \\
\text { Maximum } \\
\text { Depth, cm }\end{array}$} \\
\hline & & & & Mean & Maximum & & \\
\hline \multicolumn{8}{|c|}{ Central Ridge, West } \\
\hline C1-WI & 0.73 & $0.4-7.0$ & 6.6 & 11 & 22 & 16 & 24 \\
\hline $\mathrm{Cl}-\mathrm{W} 2 a$ & 0.31 & $7.0-9.7$ & 2.7 & 11 & 10 & 19 & 31 \\
\hline $\mathrm{C} 1-\mathrm{W} 2 b$ & 0.27 & $9.9-12.2$ & 2.3 & 12 & 22 & 17 & 22 \\
\hline C1-W3 & 0.47 & $13.8-20.5$ & 6.7 & 7 & 40 & 21 & 15 \\
\hline C1-W4 & 0.40 & $20.5-26.0$ & 5.5 & 7 & 44 & 22 & 37 \\
\hline C1-W5 & 1.31 & $26.5-34.4$ & 7.9 & 17 & 48 & 22 & 12 \\
\hline \multicolumn{8}{|c|}{ Central Ridge, East } \\
\hline C1-E1 & 1.15 & $0-9.4$ & 9.4 & 12 & 22 & 3 & 23 \\
\hline C1-E2 & 0.73 & $9.4-14.3$ & 4.9 & 15 & 26 & 8 & 34 \\
\hline $\mathrm{C} 1-\mathrm{E} 3 a$ & 0.39 & $16.1-18.4$ & 2.3 & 17 & 24 & 23 & 19 \\
\hline C1-E3b & 1.31 & $18.4-23.2$ & 4.8 & 27 & 46 & 18 & 26 \\
\hline C1-E4 & 0.46 & $27.5-31.9$ & 4.4 & 11 & 19 & 27 & 0 \\
\hline \multicolumn{8}{|c|}{ Harry's Ridge } \\
\hline H2-Rt66 & 4.54 & $0-6.6$ & 6.6 & $69^{\circ}$ & 96 & 20 & 37 \\
\hline \multirow{2}{*}{\multicolumn{8}{|c|}{ Johnston Ridge }} \\
\hline & & & & & & & \\
\hline $0 \mathrm{~m}$ & $\mathbf{0}$ & $-2.2-13.2$ & 15.4 & 0 & $\mathbf{0}$ & 25 & 0 \\
\hline $10 \mathrm{~m}$ & 8.24 & $-4.0-10.2$ & 14.2 & 58 & 110 & 24 & $\mathbf{0}$ \\
\hline $15 \mathrm{~m}$ & 11.32 & $-5.8-10.0$ & 15.8 & 72 & 120 & 26 & 0 \\
\hline $20 \mathrm{~m}$ & 7.68 & $-5.2-12.1$ & 17.3 & 44 & 90 & 30 & 0 \\
\hline $37 \mathrm{~m}$ & $\mathbf{0}$ & $-5.5-9.5$ & 14.9 & 0 & 0 & 29 & $\mathbf{0}$ \\
\hline
\end{tabular}

preblast topography, such as tree stumps or concavities, may act as nuclei for the formation of longitudinal vortices, that is, vortices aligned in the direction of flow. The nature of the preblast landscape and of its erosion during the blast can be determined by tracing individual stratigraphic units under the furrows. Figure 13 shows a cross section of one furrow (C1W3) in which uneroded contacts between two units of Pine Creek deposits ( $P$ for clay-rich ash; $\mathbf{P g}$ for a sandy ash), and Smith Creek deposits (Y) [Crandell and Mullineaux, 1973; Mullineaux et al., 1975] on the downhill side of the furrow suggest that the furrow formed where a depression had existed in the preblast topography.

Figure 14 shows four cross sections of furrowed areas in the distal zone (i.e., at localities $\mathrm{C} 1, \mathrm{H} 2$, and $\mathrm{J} 2$ ) from which estimates have been made of the amount of material removed to form furrows. In plotting the data for furrows C1-W1 to C1-W5 (Figure 14a), adjustment has been made for the abovementioned preexisting curvature of the slope by using the uneroded stratigraphic contacts as straight baselines, relative to which the measured thicknesses are indicated. The crosssectional areas of the furrows, representing the material eroded by the furrowing process, were calculated after this transformation was made. The values so obtained were as much as $50 \%$ less than before the correction, where the preexisting concavity is counted as part of the furrow. The preblast contacts observed in furrows C1-E1 to C1-E4 on the east side of the central ridge showed no such large-scale concavities, but only small-scale fluctuations, shown in Figure 14b, caused by possible inaccuracy of the measurements, bioturbation, etc. Thus a similar correction was not necessary for Figure $14 b$. Figure $14 c$ shows the cross section of perhaps the deepest furrow on Harry's Ridge, H2-Rt66 (see Figure 8). Removal of May 18 blast deposit shows that the furrow is considerably deeper than superficial examination would suggest and that the bottom actually forms a trough. Figure $14 d$ shows five cross sections (two of which are simply representations of the mean slope at the ends) of furrow J2-I5 on Johnston Ridge. As described above, this furrow and its neighbors contained no blast deposit, so no trench was dug. Cross-sectional area, width, and the average and maximum observed depth (with the blast deposit removed) derived from Figure 14 are presented in Table 2. The uncorrected slope from tangent to tangent across each furrow at the location of the traverse and the maximum depth of the blast deposit observed in each furrow are also given. The column headed "slope distance" defines the crest-to-crest limits of each furrow, in relation to the axes presented in Figure 14. The values in the column headed "slope width" are elsewhere in this text referred to as "transverse wavelength." The last five entries in the table are from the cross sections of Figure 14d.

The average depth of pre-1980 material removed from the measured furrows on the west side of the central ridge is 11 $\mathrm{cm}$, on the east side is $16 \mathrm{~cm}$, and at H2-Rt66 is $69 \mathrm{~cm}$. The total volume removed from furrow J2-I5 is approximately 200 $\mathrm{m}^{3}$ over a projected area of $520 \mathrm{~m}^{2}$, yielding a mean depth of $40 \mathrm{~cm}$. The average of this depth with the depths at localities $\mathrm{C} 1$ and $\mathrm{H} 2$ reported in Table 2 is $19.7 \mathrm{~cm}$. The transverse wavelength (slope width) ranges from about 2 to $17 \mathrm{~m}$; most often the wavelengths are in the range $3-7 \mathrm{~m}$. Treating $\mathrm{C1}$ W2a,b and C1-E3a,b each as one furrow and counting the mean slope width of J2-I5 once, the furrows in Table 2 have mean slope width of $7 \mathrm{~m}$. (We note that these dimensions are 


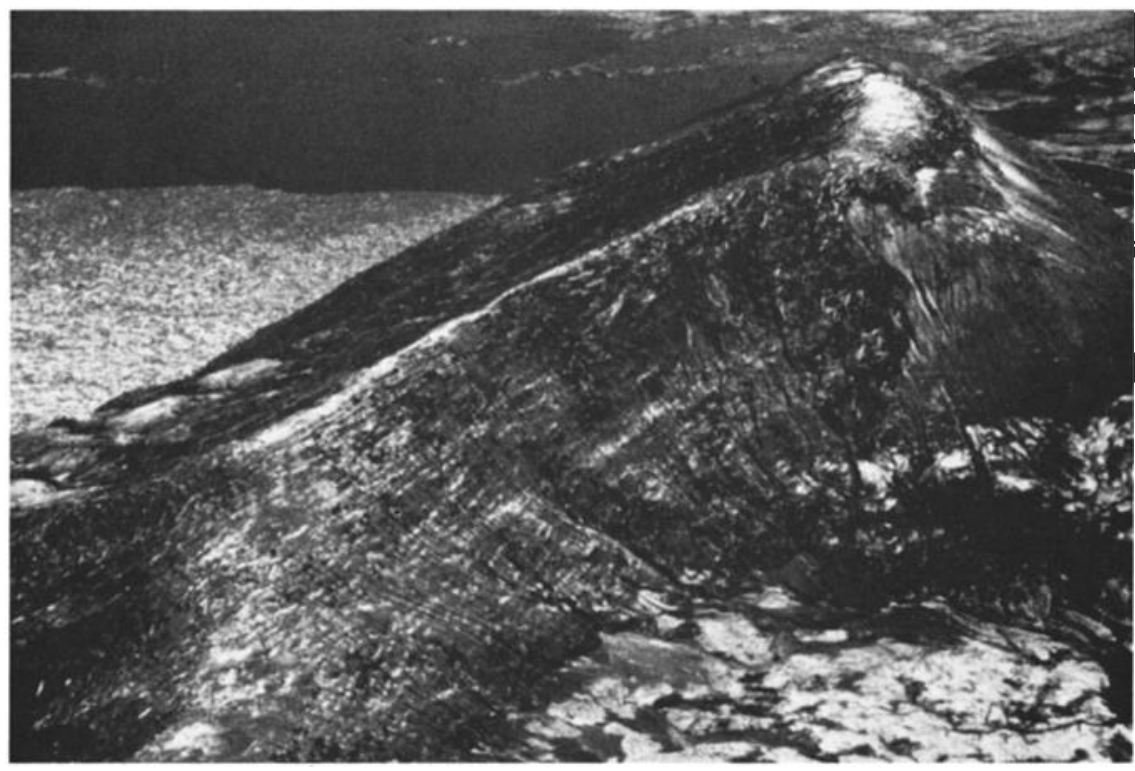

Fig. $7 a$

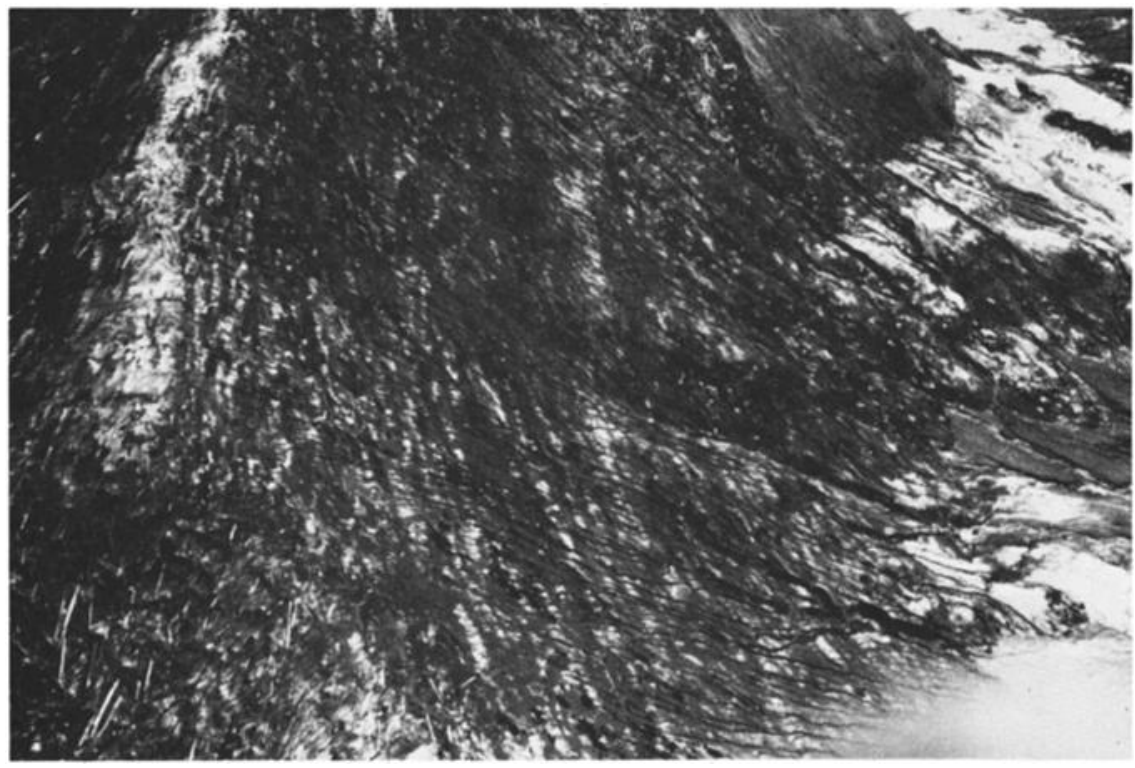

Fig. $7 b$

Fig. 7. (a) Aerial photo of the headwall of South Coldwater Creek and summit of Harry's Ridge with furrows from center to lower left appearing light due to ponding of deposits. View is toward the southeast. At center right, directly below summit ridge on the west slope, is the sheltered region (with downed trees) from which the furrows originate. Another sheltered slope, extreme left, on the east side of the ridge terminates the furrows. White region is avalanche deposit, covered by pyroclastic flows. Bare zone in the right background is the avalanche scar (or secondary landslide) on the west side of Harry's Ridge. Many of the furrows in this photo are indicated in Figure 4, west of the ridge crest. Straight-line distance from the ridge summit to the end of the ridge at the bottom of the photograph is $800 \mathrm{~m}$. (b) Enlarged view, from a more vertically oriented vantage point, of the furrowed region of Figure $7 a$, showing the high contrast of blast deposits in furrows. A multitude of erosional gullies (dark sinuous lines angling down and to the right) forms a grid almost orthogonal to the furrows (white markings angling up and to the left). Many of the furrows in this photo are indicated in Figure 4, west of the ridge crest. Distance along light-colored ridge crest from the top of photo is $240 \mathrm{~m}$.

similar to the typical spacing between Douglas fir trees.) Furrow lengths are often $100 \mathrm{~m}$, but furrows with lengths only about twice their width do occur (e.g., J2-I5; see Figure 3).

Furrows that cover a large area seem to merge and/or bifurcate at their ends, so in places, paths of furrows may be followed for several hundred meters, especially at Harry's Ridge.
Because of the variability introduced by the merging and shifting of the furrows, it is difficult at any given site to discern an increase in their lateral spacing with distance from the source (for example, as would be expected from downstream growth of vortices within the flow).

The mean depth of the furrows ranges from 7 to $72 \mathrm{~cm}$, with 


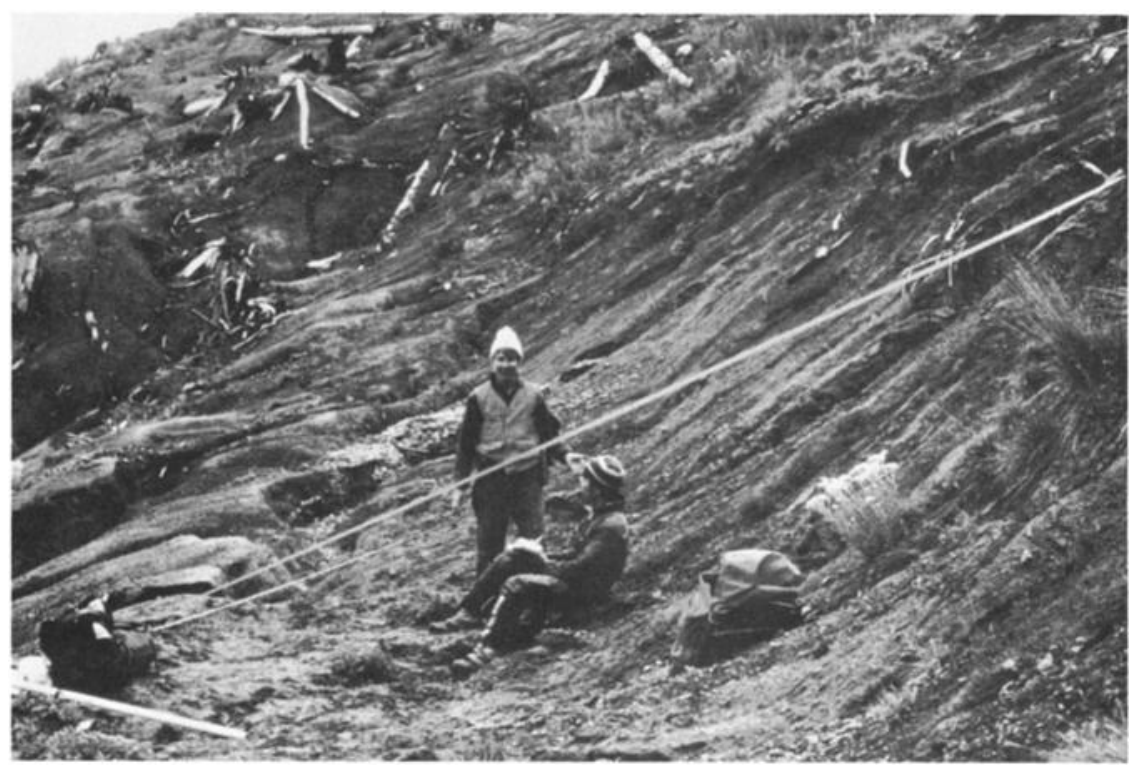

Fig. 8. Furrow H2-R166 which originates downstream of a small sheltered region on the east side of Harry's Ridge (locality H2). The region of tree trunks in the upper center of the photo is the sheltered separation zone. The furrow begins at the reattachment point at the edge of this separation zone and extends $100 \mathrm{~m}$ downstream (behind the photographer). The straight tape is stretched from one edge of the furrow to the other, thus showing the inferred original slope. Note the prominent zone of soil and roots on the uphill edge of the furrow. The land surface was everywhere lowered at least to the level of these tree roots and was additionally lowered within furrows such as this one. An erosional gully starts at the top right behind the standing figure, turns down to the bottom of the furrow, where it runs along the furrow until it cuts through the lower crest (at the base of the tape) into the next furrow downhill. The visible portion of the tape is $6.1 \mathrm{~m}$ long. See also Figure $14 c$.

many being of the order of $20 \mathrm{~cm}$ deep. On the other hand, as many as $30-50 \mathrm{~cm}$ of soil above the remaining crest lines of the furrows (that is, above the small Douglas fir roots that are exposed on the tops of these crests) may also have been removed. Thus the currently exposed furrows could represent as little as one third of the total amount of material eroded by the mechanism of erosion responsible for the furrowing.

\section{Fluid-Mechanic Origins OF THE FURROWS}

The implications regarding the nature of the blast flow field suggested by the scale and regularity of the furrows at Mount St. Helens are significant. The occurrence of regular structures in a flow at Reynolds number as high as that of the blast (see section 4) indicates that there was strong coupling and feedback between the topography, the process of furrow formation, and the flow itself. Such feedback and coupling lead to the development of large-scale vortical motions in turbulent shear flows. These organized motions contain much of the turbulent energy and occur at the largest scale of the shear flow, i.e., of the boundary layer thickness (the boundary layer is defined to be the region of the blast flow adjacent to the ground in which deceleration by shear stresses on the ground occurred). One such motion which is frequently observed is a periodic array of large vortices aligned with the flow direction. These enhanced motions may originate from, but are distinctly different from, the much smaller, more transitory and irregular longitudinal vortices that are observed in the viscous sublayer of turbulent boundary layers, to which the formation of sand streaks has been attributed [Weedman and Slingerland, 1985]. As already stated, we assume that the furrows at
Mount St. Helens were produced by such longitudinal vortices. The swirl velocity at ground level under such vortices can be of the same order as the free-stream velocity outside the boundary layer. Because of this high velocity, as well as the fact that the pressure at the eye of the vortices might be quite low, adding a vacuuming effect, erosion under the vortices can be much larger than in a vortex-free flow. Evidently the vortices formed shortly after tree removal (early in the erosive phase of the blast flow) and then were maintained long enough to erode into the furrows. It is likely that local vortexgenerating features, such as tree stumps and small topographical depressions, determined the specific sites at which the vortices nucleated. Similar processes involving longitudinal vortices are thought to be the cause of spiral grooves and crosshatching which develop on supersonic reentry bodies [Canning et al., 1968; McDevitt and Mellenthin, 1969; Tobak, 1969].

Large-scale vortical motions in turbulent shear flows are generated by flow instability. Longitudinal vortices in wallbounded flows are observed in two different fluid-mechanical settings, corresponding to the two settings described for Mount St. Helens in section 2 (Figures 1 and 4). In the first setting, a separated free shear layer dividing high-speed outer flow from inner, low-speed recirculating flow reattaches to a wall. Centrifugal instabilities in the shear layer upstream of reattachment [Konrad, 1976; Bernal and Roshko, 1986] and in the flow that is turning parallel to the wall in the neighborhood of reattachment [Ginoux, 1958] generate streamwise vorticity. The pertinent instability mechanism was treated by Taylor [1923] and Görtler [1954], the latter in the context of boundary layer flow over a concave surface. The instability 
acts independently of the action of viscosity. Low-energy fluid near the wall, if displaced away from the wall toward the center of curvature of the wall, tends to "fall" toward the center of curvature, while displacement of high-energy outer fluid toward the wall causes it to spin away from the center of curvature toward the wall. In both cases the initial displacement increases, signaling instability. The Taylor instability leads to the development of an array of counterrotating pairs of longitudinal vortices and is the cause of the transition from laminar to turbulent flow on concave plates. Streamwise vortices are observed in similar circumstances in both laminar and fully turbulent flow, and they occur in both subsonic and supersonic flow [Tani, 1962; Ginoux, 1958]. In particular, Ginoux showed that they occur downstream of reattachment behind backward facing steps in supersonic flow. Hopkins et al. [1960] used improved flow visualization to highlight the vortices (Figure 15a). The apparatus in this experiment can be compared with the geometry at Harry's Ridge shown in Figure 4, and the oil streaks downstream of the backward facing step with the furrows on Harry's Ridge (Figure 7a).

In the second setting, the component of flow normal to the long axis of slender bodies (for example, cones) at small angle of attack is unstable [Gregory et al., 1955; Stuart, 1963]. (Angle of attack is defined as the angle between the direction of the undisturbed oncoming flow and the axis of a body embedded in the flow.) The cross-flow instability occurs in low Reynolds number laminar and transitional boundary layers; its development depends on the existence of viscous stresses in the layer, and it is similar to the Tollmien-Schlichting instability [Tollmien, 1929; Schlichting, 1933] in plane flow over a flat plate. Cross-flow instability waves form normal to the cross-flow velocity component and so, when fully developed, appear as an array of corotating vortices aligned very nearly in the streamwise direction. Figure $15 b$ shows the ablation pattern formed by streamwise vortices on a $10^{\circ}$ half-angle cone of $\mathrm{NH}_{4} \mathrm{Cl}$ tested at $10^{\circ}$ angle of attack in air flowing at Mach number $M=7.4$. It is presumed [McDevitt and Mellenthin, 1969] that the appearance of corotating vortices in high Reynolds number turbulent boundary layers is also due to the cross-flow instability.

The central ridge of Spirit Lake is very similar to a half cone; from map measurements, the semivertex angle $\theta$ can be determined to be approximately $16^{\circ}$. The long axis (crest) of the ridge lies at an "angle of attack" $\alpha$ of about $15_{-0}^{\circ+3}$ to the direction from the presumed source of the blast in the mouth of the present crater. The two cones in Figure $15 b$ are oriented to simulate the plan view and the elevation view of the central ridge locality, respectively. Spirit Lake provides a plane of symmetry about which the central ridge would be "reflected" to complete a full three-dimensional conical shape. Although the geometry of the Studebaker Ridge locality is not as simple as that of the central ridge, there was a component of cross flow there as well, and we suggest that the furrows at Studebaker Ridge result from the same flow mechanisms as at the central ridge.

Longitudinal vortices occur in both subsonic and supersonic flow. Laboratory experiments on supersonic reattaching flow [Ginoux, 1958] suggest that they are stronger and more regular than in subsonic flow. It is not known whether the development of streamwise grooves on a body downstream of reattachment further intensifies and organizes the longitudinal vortices. On the other hand, the formation of ablation pat-

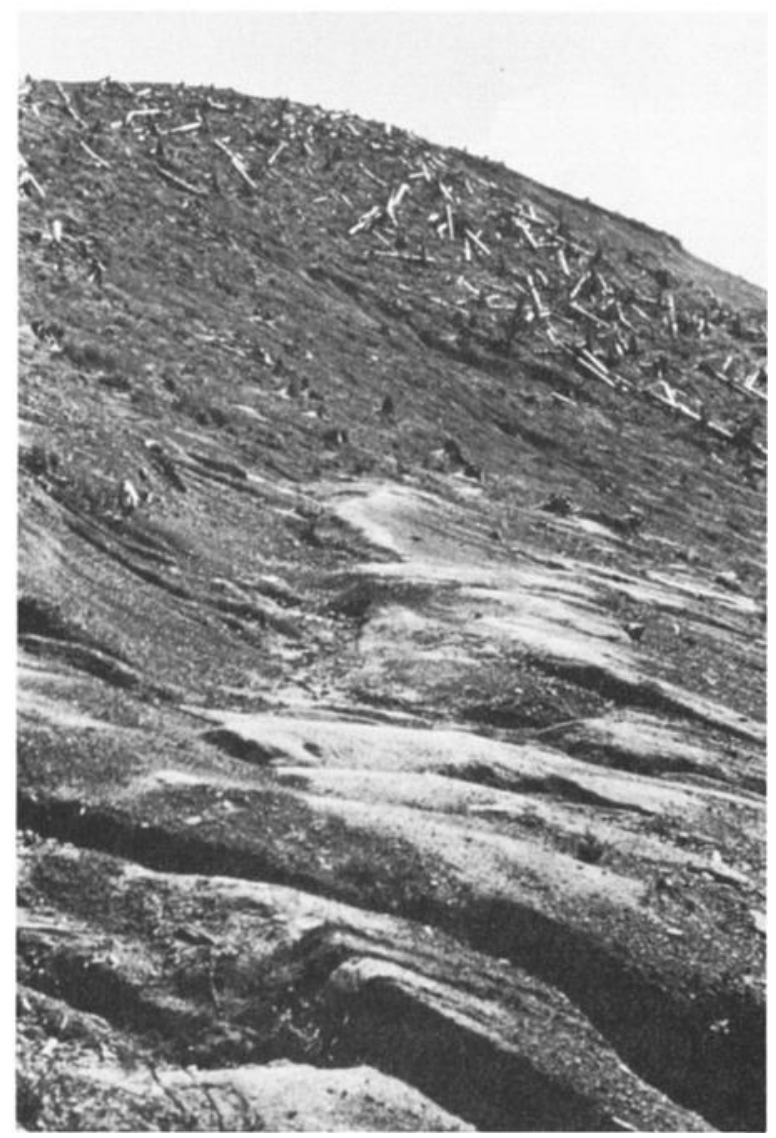

Fig. 9. Looking upstream along the axis of a furrow toward the topographically sheltered zone on Harry's Ridge. The view is to the south, and the crest of the ridge shows at the top. The furrow is expressed as the trough at the left of the photograph; it is partially filled with blast deposits toward the right. These deposits have been cut by water running down the axis of the furrow (toward the camera) and then downslope to the right. This fluvial gullying produces the pseudo-dune form of the blast deposit that filts the furrow. The zone of downed trees and small vegetation toward the top of the photo is the sheltered zone, where the flow separated as it came over the ridge toward the viewer. Note the decrease in the height of standing trunks toward the observer. The decrease in these stump heights was used to obtain the estimate of radius of curvature for flow reattachment of $100 \mathrm{~m}$ in section 4 of the text. Some of the larger stumps at the top of the separation zone are shown in Figure 10. The flow reattachment point is at the vegetation line (this scrub vegetation was presumably protected from erosion and furrowing by snow cover). The distance from the skyline ridge to the photographer is about $260 \mathrm{~m}$.

terns due to cross flow on slender bodies depends on waves induced by the altered surface geometry. There are only two situations in which such waves can occur. In one, the flow relative to the body is supersonic, and standing waves lie in the supersonic region between the body and the shock wave that wraps around the body [Tobak, 1969]. In the second, a thin layer of dense fluid moves at supercritical velocity over the body, and the waves stand on the free surface of the supercritical flow [Persen, 1971]. The formation of sand waves is a well-known example of the latter process [Simons et al., 1965]. Thus the furrows at Mount St. Helens may be taken as tentative evidence of either (1) supersonic velocity in a relatively deep blast flow or (2) supercritical flow in a shallow density current. Unfortunately, little theory is available to provide the 


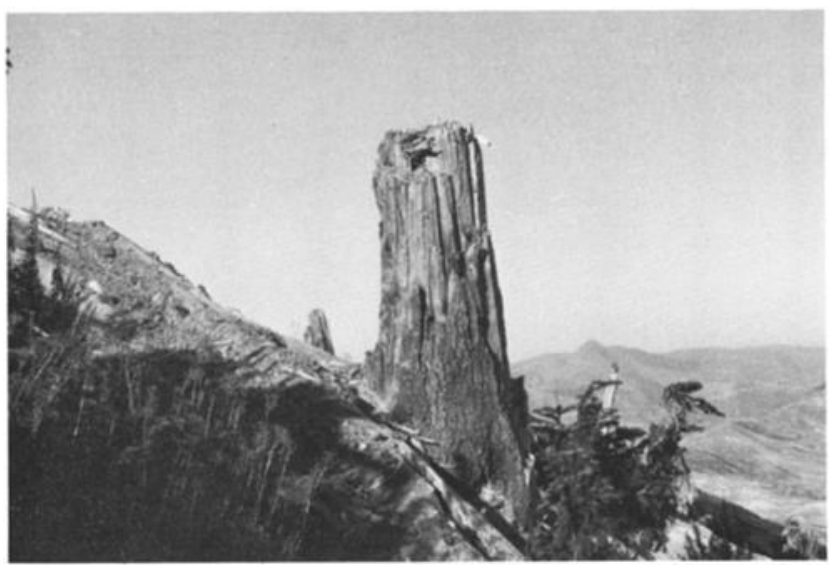

Fig. $10 a$

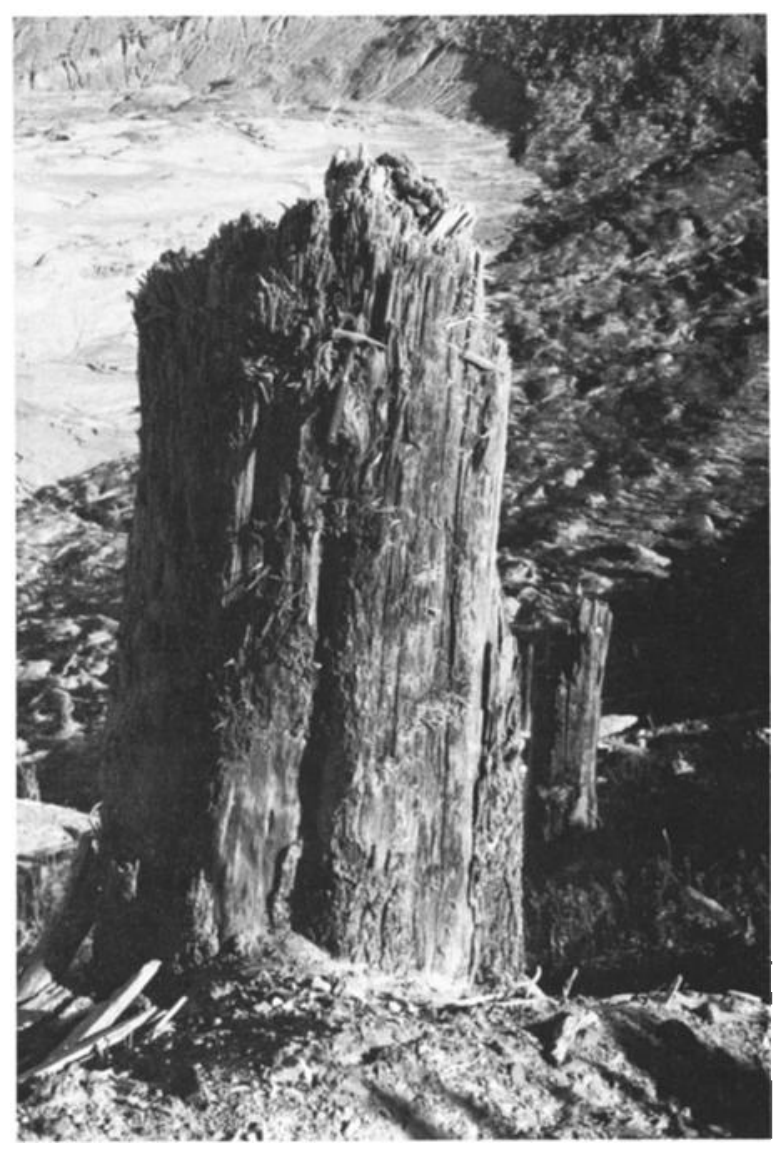

Fig. $10 b$

Fig. 10. Evidence for recirculation of flow in the protected region shown in Figure 9. (a) A sheared tree stump viewed orthogonal to the blast flow direction (left to right). In contrast to the "war bonnet" shape typical of many stumps at other locations in the devastated area [see Kieffer, 1981, p. 382], this stump has a more conical shape. The uphill side of the trunk is about $1.2 \mathrm{~m}$ high. (b) The upstream side of the same stump showing abrasion and pitting on the scale of a few centimeters diameter. (c) The downstream abrasion and pitting of the same stump, indicating reverse flow, but erosion by much smaller particles than in the direct flow, implying a lower velocity in the recirculating flow. (d) A conical stump typical of the protected region, indicating roughly equal abrasion from all directions. The stump is approximately $3 \mathrm{~m}$ tall.

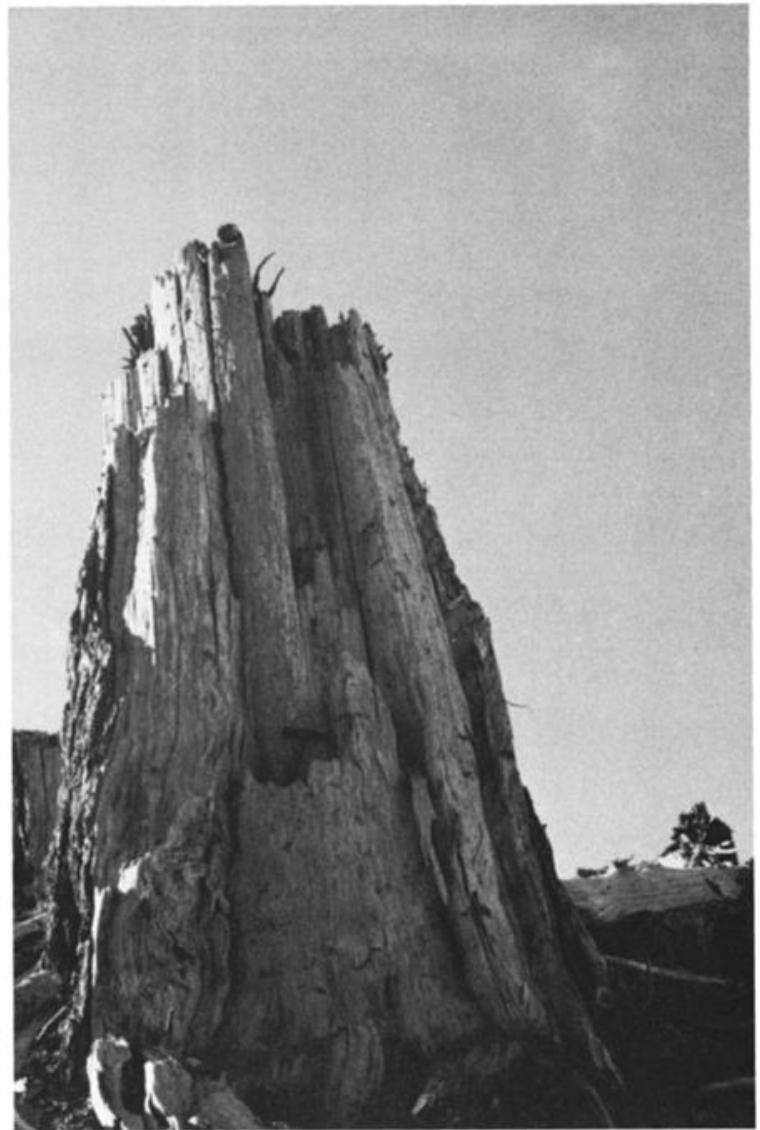

Fig. $10 c$

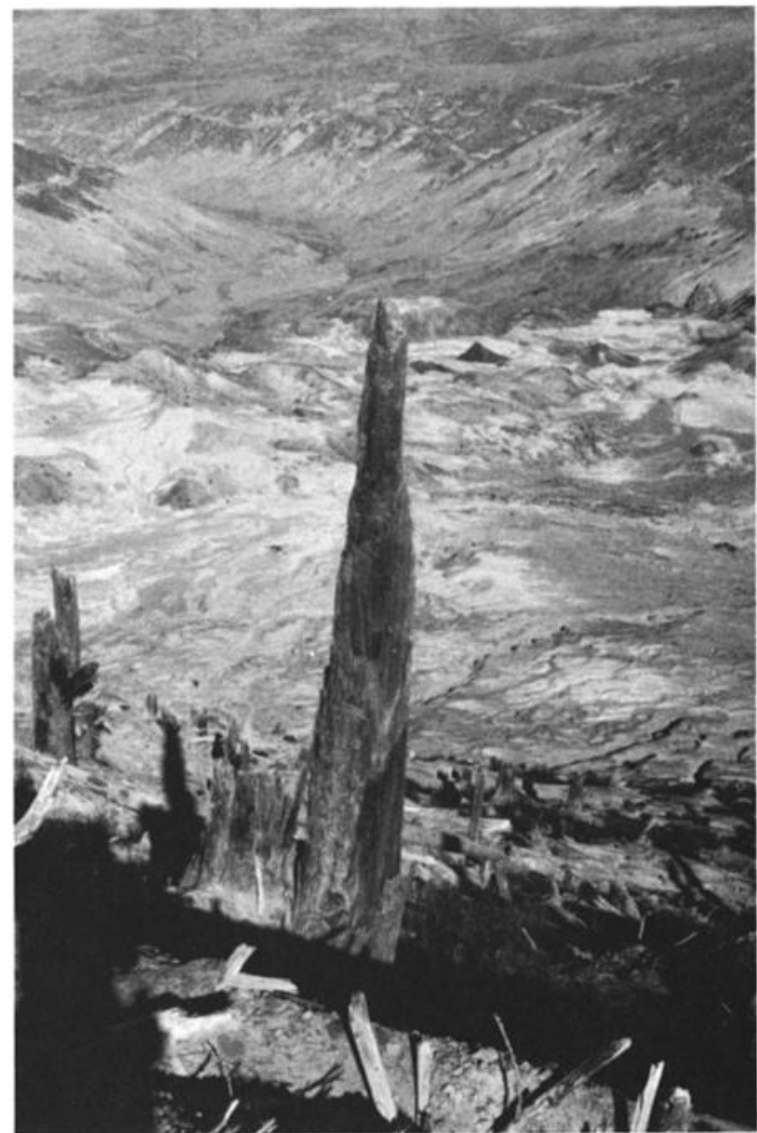

Fig. 10d 


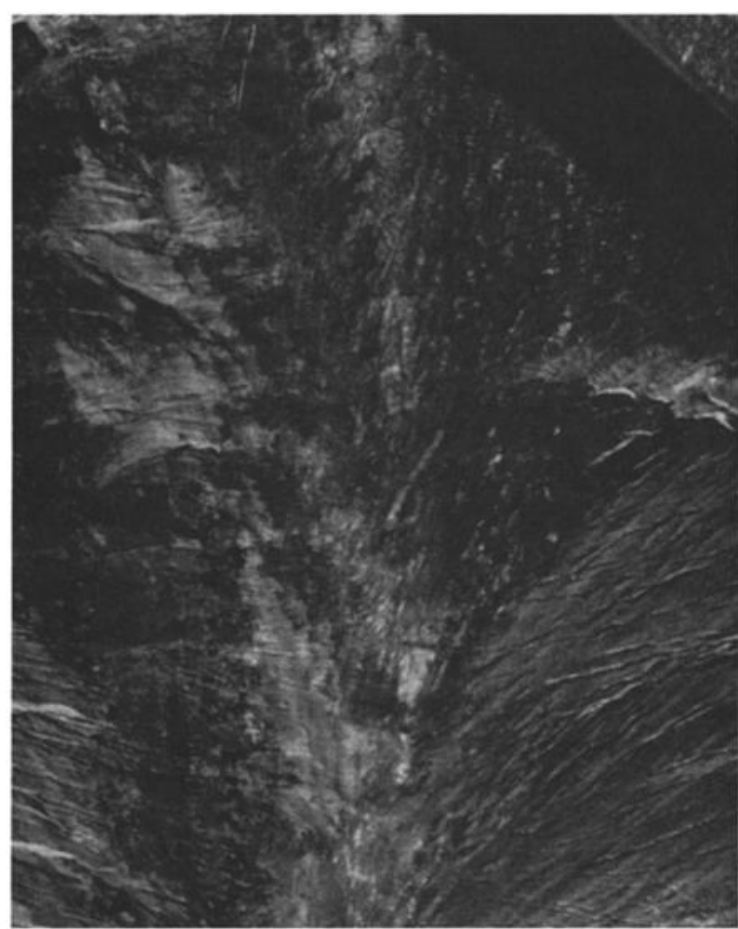

Fig. $11 a$

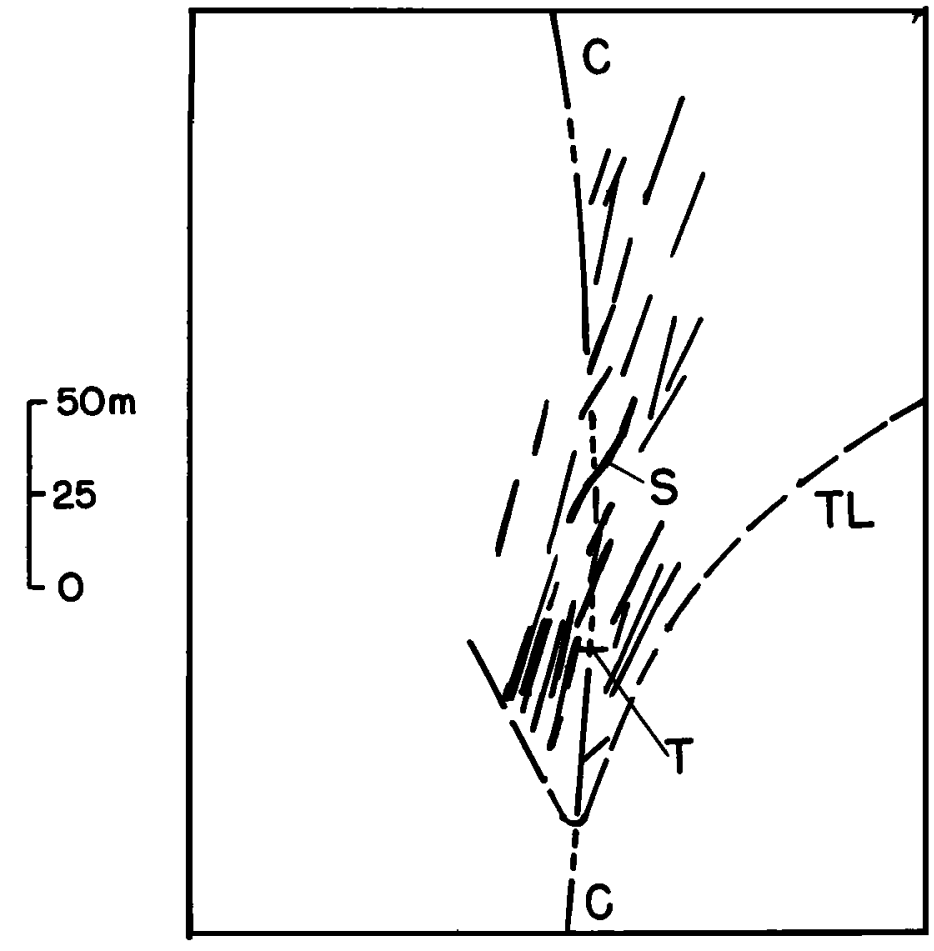

Fig. $11 b$

Fig. 11. (a) Aerial view from a point directly above the crest of the central ridge, showing a subtly S-shaped furrow crossing the crest from left to right at the center of the photo. North is toward the top of the photo. Throughout the top two thirds of the photo, furrows are seen crossing the crest at angles ranging from $11^{\circ}$ to $33^{\circ}$. The trench through furrows C1-E1 to C1-E4 shows as a dark horizontal mark at the north end of the gray patch lying halfway between the S-shaped furrow and the bottom of the photo. The arc running from bottom center to center right delineates the limit (trim line), $140 \mathrm{~m}$ above the present level of Spirit Lake, of the run-up of the wave in Spirit Lake generated by the debris avalanche on May 18, 1980. (b) Schematic illustration of some of the furrows in Figure 11a. S, S-shaped furrow; T, trench; TL, trim line; and $\mathrm{C}$, ridge crest.

basis for testing the second hypothesis; below we discuss the former rather extensively.

It is clear that the swirling motion in longitudinal vortices can bring high-energy fluid to the ground, thereby increasing shear and erosion. The question arises whether the flow in counterrotating vortices generated by the Taylor mechanism might be different enough from the flow in corotating crossflow vortices that erosional differences which permit the distinction of the two mechanisms would be visible. Since at ground level the swirl velocities of neighboring counterrotating vortices are in opposition, while those of corotating vortices are always in the same direction, it is possible that the former would tend to erode cross sections with sharper crests than would the latter. We have noticed that the furrows at Studebaker Ridge and the central ridge are distinctly smoother in appearance than those that occur downstream of sheltered regions (cf. Figures 2 and $5 a$, and $6 a$ and 3, respectively). This observation would be consistent with furrows at these different locales originating from the two different mechanisms described above.

\section{Implications of OBSERVATIONS}

In this section we express our results in a form which permits comparison to other observations, and we estimate some properties of the highly erosive initial stage of the blast. Erosion rates in turbulent flow are commonly associated with shear stresses exerted on the eroding surface by the moving fluid in the boundary layer. The shear, in turn, depends on properties of the flow further away from the surface, such as the free stream flow velocity $U$, flow density $\rho$, viscosity $\mu$, and also on the boundary layer thickness $\delta$. Indeed, the rate at which momentum is added to or extracted from the flow by shear, and also the rate of mass and energy transfer caused by erosion, deposition, drag, and heat transfer at the surface of the ground, all determine the local growth rate of the boundary layer at any station downstream from the source of the flow. Thus if we could measure the rate of boundary layer growth by assuming, say, that an observed increase of furrow spacing in the streamwise direction at a given locality indicates an increase of vortex diameter and therefore of boundary layer thickness, we could obtain significant information about transport. Unfortunately, as already noted, because of the variability introduced by the merging and shifting of furrows, it is difficult at any given site to discern an increase of furrow spacing in the streamwise direction. On the other hand, the absolute magnitude of the boundary layer thickness, which is determined by the accumulated history of the flow from its origin to the point of observation, is rather well constrained by the observations of furrow spacing, so an indirect estimate of the erosional efficiency can in fact be made. In addition, we use the orientation of the furrows near the crest of the central ridge as a measure of the "upwash angle" there to obtain an estimate of the Mach number and flow velocity. Finally, we discuss the implications of the observed boundary layer thickness and compare the erosive efficiency of the furrow-making process with that of laboratory-observed vortex-free flows. 


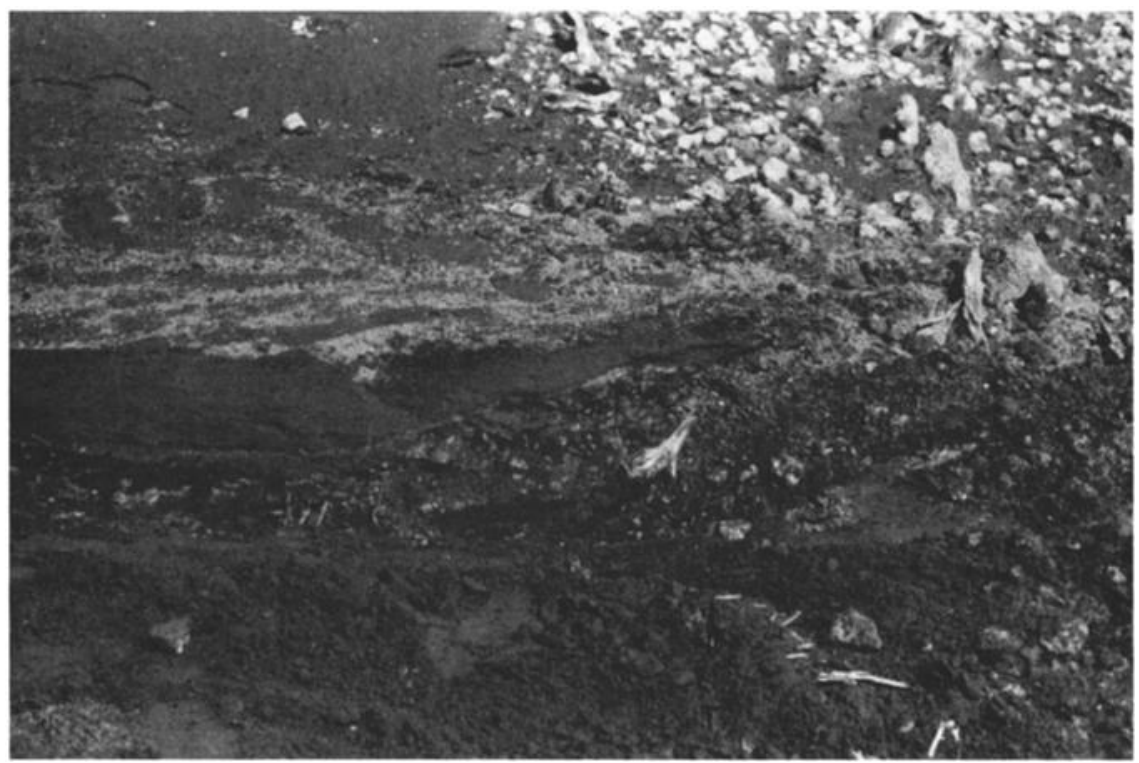

Fig. $12 a$

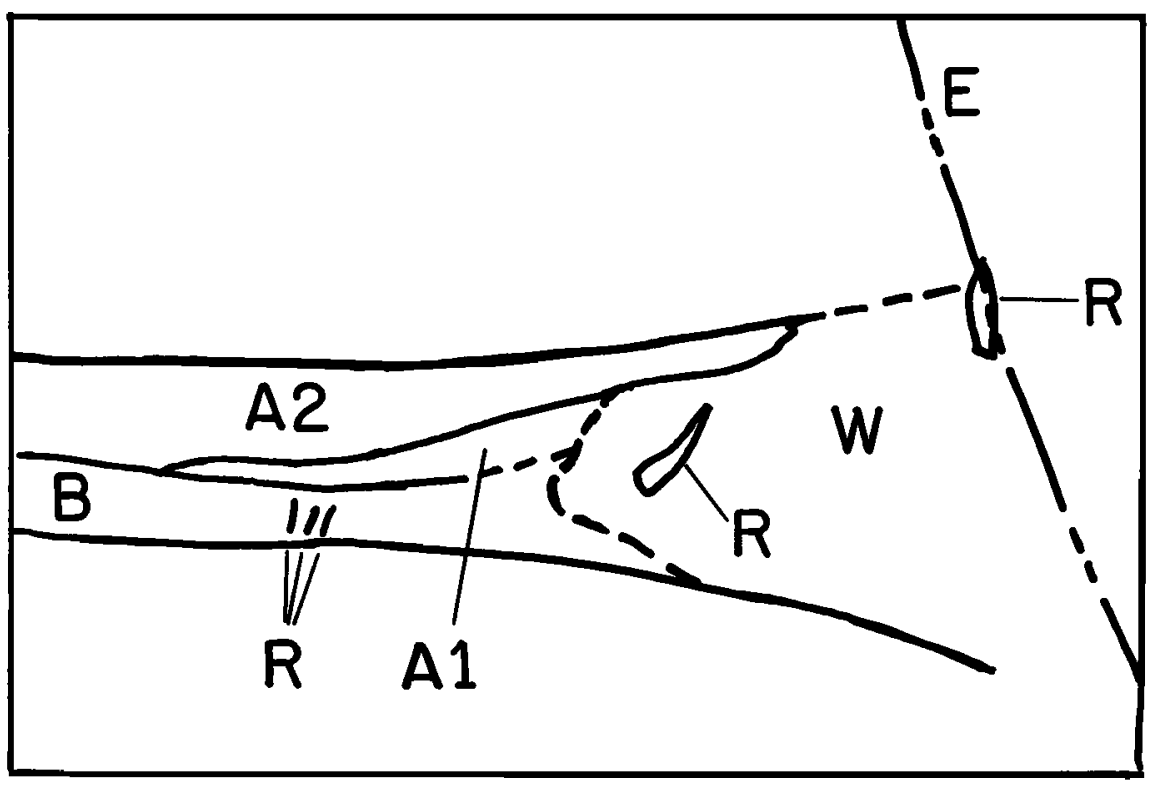

Fig. $12 b$

Fig. 12. (a) Photograph of the downhill edge of a trenched furrow (C1-E1) at the crest of the Central Ridge, with schematic illustration of features in Figure $12 b$. The view is downstream along the furrow axis. The trench is orthogonal to the axis. The furrow extends into coarse $W$-pumice on the right, where remnant tree root fragments $(R)$ are visible. The lens-shaped deposit is lateral blast material filling the furrow (the deposit is largely the fine-grained layer 2 (A2), but coarse layer 1 (A1) material is visible at the base of the blast deposit). The furrow has been eroded through W-deposits into underlying Castle Creek B deposit (dark brown scoria) (B) and (not shown) P deposits (fine, light brown-pink ash). Terminology of deposits from Crandell and Mullineaux [1973]. In Figure $12 b$ the line E indicates the projected edge of the furrow into the third dimension, the downstream flow direction. The furrow is the contact between the blast units A2 or $A 1$, and units $B$ or $W$.

The discussion in this section is premised on the assumptions of the single-fluid theory of equilibrium dusty gases [Marble, 1970], in which momentum and energy exchange between the phases occur rapidly and the solid particles do not interact. Under these assumptions the flow density is given by the sum of the material density $\rho_{v}$ of the vapor phase and the distributed density $\rho_{s}$ of the solid phase, $\rho=\rho_{v}+\rho_{s}$, while the viscosity is simply the material viscosity of the vapor phase, $\mu=\mu_{v}$. If the particles are not of uniform size, they do not interact only if the total volume they occupy is small, i.e., $\rho_{s} \ll d_{s}$, where $d_{s}$ is the material density of the solid material. The values of $\rho_{v}, d_{s}$, and $\mu_{v}$ are relatively well constrained for explosive eruptions of the magnitude of the May 18, 1980, eruption of Mount St. Helens. Using conditions appropriate to the regions in which furrows are observed, we take the density and viscosity of hot steam at 1 atm and $220^{\circ} \mathrm{C}$ to be 


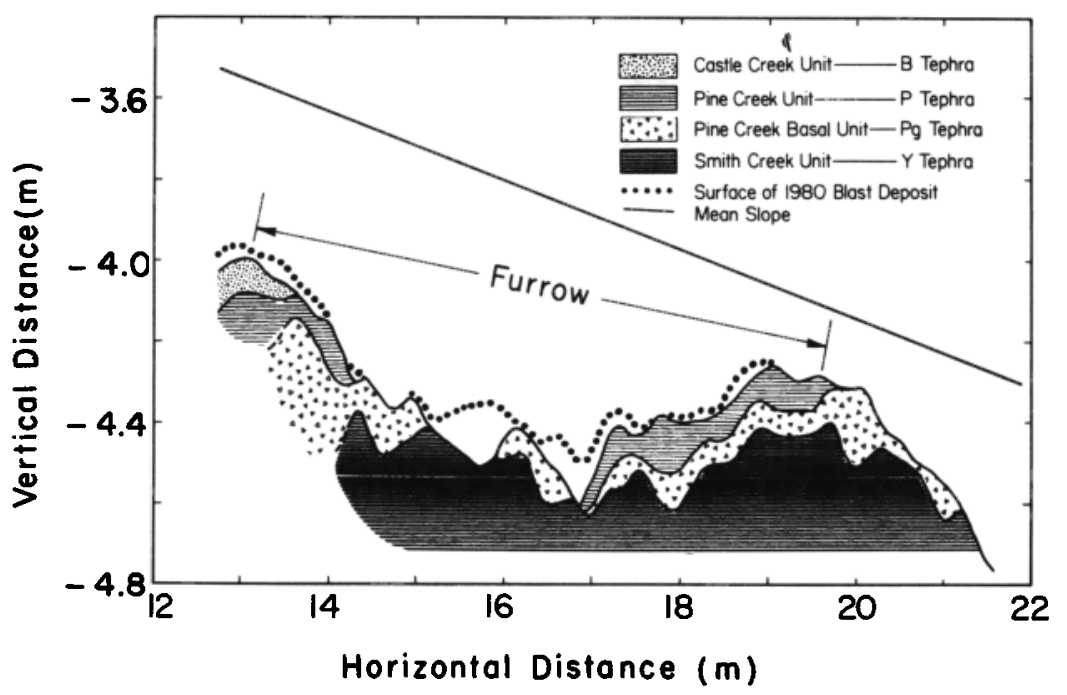

Fig. 13. Cross section of furrow C1-W3, the third furrow below the crest on the west side of the central ridge into Spirit Lake. The vertical scale is magnified $5.4 \times$, and distances from a baseline sloping at the actual mean slope of the hillside (solid line) are plotted in the figure. The origin of the vertical and horizontal coordinates is at a reference point at the upslope extremity of the traverse; the labels on the vertical axis apply to the cross section at the left edge of the plot (horizontal distance $12.7 \mathrm{~m}$ ). On the downhill side of the furrow there are two uneroded depositional contacts $(\mathrm{Pg} / \mathrm{Y}$ and $\mathrm{P} / \mathrm{Pg}$ ) which depart significantly from the mean slope of the hill. If the original surface of the hill followed these contacts (as might be expected by mantling ash deposits), then it could be inferred that the slope was locally slightly concave at this location. (The magnification of the vertical distance below the sloping baseline exaggerates the curvature; a local elevational minimum does not actually occur.) It is likely that such concavities, if extensive enough in the fow direction, could act as nuclei for the formation of longitudinal vortices.

representative of the vapor phase, namely, $\rho_{v}=0.44 \mathrm{~kg} / \mathrm{m}^{3}$ and $\mu_{v}=1.7 \times 10^{-5} \mathrm{~kg} \mathrm{~m}^{-1} \mathrm{~s}^{-1}$, and for the solid phase we take $d_{s}=1300 \mathrm{~kg} / \mathrm{m}^{3}$. On the other hand, the flow velocity, flow time $t$, and flow density are very poorly constrained. $U$ may be as small as 10 or as large as $500 \mathrm{~m} / \mathrm{s}$, while in the regions where furrows occur, plausible values of $t$ range from 10 to $100 \mathrm{~s}$, and $\rho$ from 0.1 to $500 \mathrm{~kg} / \mathrm{m}^{3}$.

For the purposes of the calculations to follow we take the boundary layer thickness $\delta$ to be twice the lateral spacing $\lambda$ of the furrows. Using the mean value of the slope width cited after Table $2(\lambda \approx 7 \mathrm{~m})$, we obtain $\delta=14 \mathrm{~m}$.

\subsection{Instability Near Reattachment: Harry's Ridge}

As stated above, there is considerable streamwise vorticity in free turbulent shear layers [Bernal and Roshko, 1986], presumably due to centrifugal instability in the large transverse rollers which comprise the layer [Brown and Roshko, 1971, 1974]. Sigurdson [1986] showed by flow visualization how streamwise vorticity in a reattaching shear layer gets intensified during the process of reattachment. The possibility also exists that additional longitudinal vorticity can be generated in the neighborhood of reattachment by the Taylor-Görtler instability, because the streamlines above the point of reattachment are curved concave up. Here we assess the plausibility of this hypothesis by estimating the radius of curvature of the flow at two different locations on Harry's Ridge.

A dimensionless form of wall curvature which measures the sensitivity of boundary layers to the Taylor-Görtler instability is the Görtler number,

$$
G=R e_{T \theta}(\theta / r)^{1 / 2}
$$

where $r$ is the radius of curvature of the flow, $\theta$ is the momentum thickness of the boundary layer, and $R e_{T \theta}$ is the turbulent
Reynolds number based on the momentum thickness,

$$
R e_{T \theta}=\frac{\rho U \theta}{\mu_{T}}
$$

$\mu_{T}$ is the turbulent eddy viscosity, which accounts approximately for increased transport in turbulent flow. According to stability theory, a finite positive value of the Görtler number of order one is required before Taylor-Görtler vortices will form. The $\theta$ measures the loss of momentum in the boundary layer due to shear stress at the surface, and its size is about $0.09 \times \delta$ [White, 1974, p. 495$]$, i.e., approximately $1.3 \mathrm{~m}$ for the blast flow at Mount St. Helens. It appears here because it is found to provide the most effective scaling of stability results [Tani, 1962]. Tani [1962] argued that for turbulent boundary layers, $R e_{T \theta}$ is approximately 43 , so

$$
G=43(\theta / r)^{1 / 2}
$$

The streamlines in the flow over Harry's Ridge are curved for two reasons: (1) due to the separation and reattachment of the flow at the prow (see Figure $4 b$ ) and (2) due to curvature of the topography along the cirque wall at the head of south Coldwater Creek (see Figure 4a). It is possible to estimate a typical flow curvature just upstream of reattachment by assuming that the heights at which trees in the sheltered zone were sheared off (sketched schematically in Figure $4 b$ ) indicate the boundary between high-speed and low-speed flow, the dividing streamline. A curve drawn through the upper ends of the remnant stumps is concave up with radius of curvature of about $100 \mathrm{~m}$. Thus the Görtler number at the reattachment point $G_{r}$ is approximately 5 , indicating that the reattachment conditions were strongly favorable to the formation of the longitudinal vortices. Fitting a circle to the curvature of the 


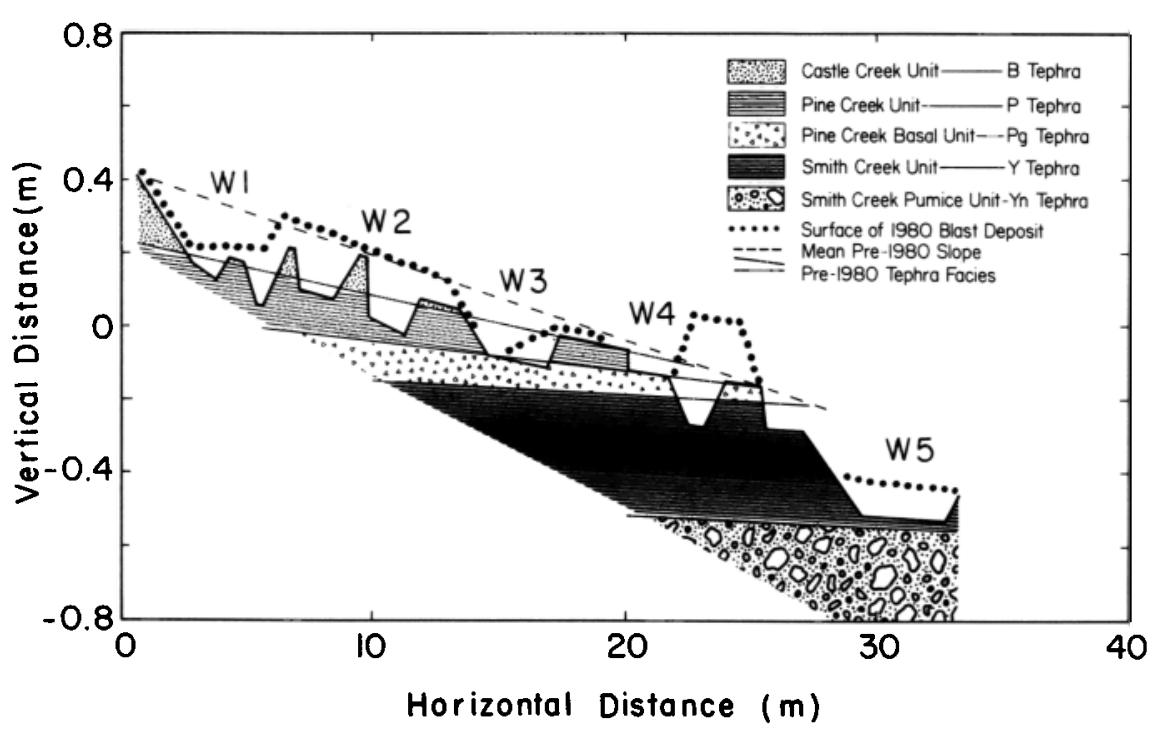

Fig. 14a

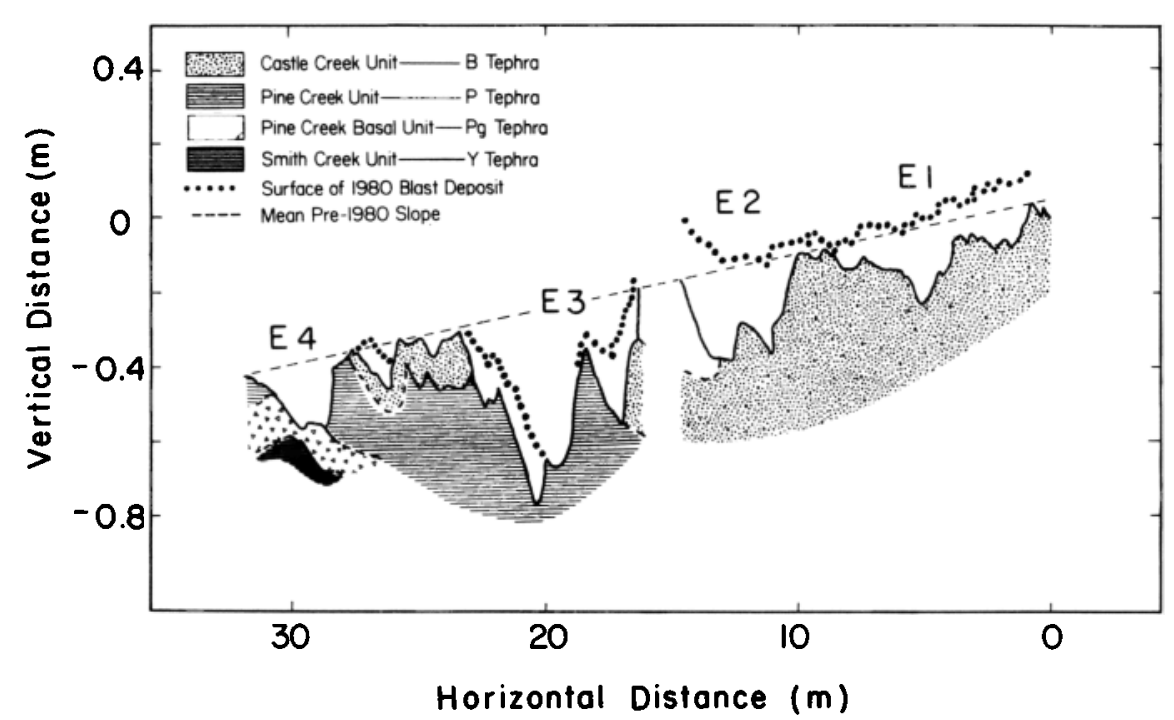

Fig. $14 b$

Fig. 14. (a) Schematic representation of furrows C1-W1 to C1-W5 on the west side of the central ridge, corrected for preblast curvature of the slope. Vertical scale magnified $14.5 \times$. The horizontal origin is the same as in Figure 13, and the vertical origin is at an arbitrary point on the B/P contact. All vertical dimensions are plotted relative to a measured or interpolated uneroded contact; $\mathrm{B} / \mathrm{P}$ for $0<x<13.8 \mathrm{~m}, \mathrm{P} / \mathrm{Pg}$ or top of $\mathrm{Pg}$ for $13.8<x<25.6 \mathrm{~m}$, and $\mathrm{Y} / \mathrm{Yn}$ or top of $\mathrm{Yn}$ for $27.6<x<33.4 \mathrm{~m}$. The plotted contacts, where not used as baselines, are smoothed to straight lines. In addition, to simplify the definition and calculation of volume of the furrows, the curvature of the slope has been removed by rotating the $\mathrm{B} / \mathrm{P}$ contact $0.53^{\circ}$ clockwise in physical shape $\left(7.7^{\circ}\right.$ on the figure) about the point $(x=15 \mathrm{~m}, y=0 \mathrm{~m})$. The changes resulting from the above transformation may be seen by comparing the profile of the furrow labeled W3 with Figure 13 . This correction procedure does not account for pre-1980 nonuniformities which may have been eroded away by the blast flow and which, depending on their curvature, would have increased or decreased the estimated cross-sectional area. Note that the $\mathrm{B}, \mathrm{P}$, and $\mathrm{Pg}$ units taper in the downhill direction. (b) Furrows C1-E1 to C1-E4 on the east side of the central ridge. In this case, no local anomalies in the preblast topography were suggested by the orientation of depositional contacts, so to simplify the definition of furrows, the curvature of the slope has been removed simply by plotting distances from baselines laid from crest to crest of each furrow. Then the furrow boundaries are defined as the points of tangency with the line of mean slope. Vertical scale magnified 14.5 x. (c) Cross section of furrow H2-Rt66. Vertical scale magnified $1.4 \times$. (d) Profile of furrow J2-15 at three axial stations (10, 15, and $20 \mathrm{~m})$, and the hill slope at each end of the furrow (0 and $37.5 \mathrm{~m}$ ). Axial distance increases in the upstream direction (south).

cirque wall, we estimate this radius of curvature to be $\mathbf{2} \mathbf{~ k m}$. Thus on the cirque downstream of reattachment the Görtler number was about 1.4, indicating that Taylor-Görtler vortices might have formed at this site even in the absence of separation. We speculate that the exceptional length of the furrows on the west side of Harry's Ridge (and to some extent, on the east side), in comparison to the short furrows found on the north side of Johnston Ridge, may be due to the fluidmechanically favorable (i.e., concave) topography downstream of reattachment at Harry's Ridge, in contrast to that at the 


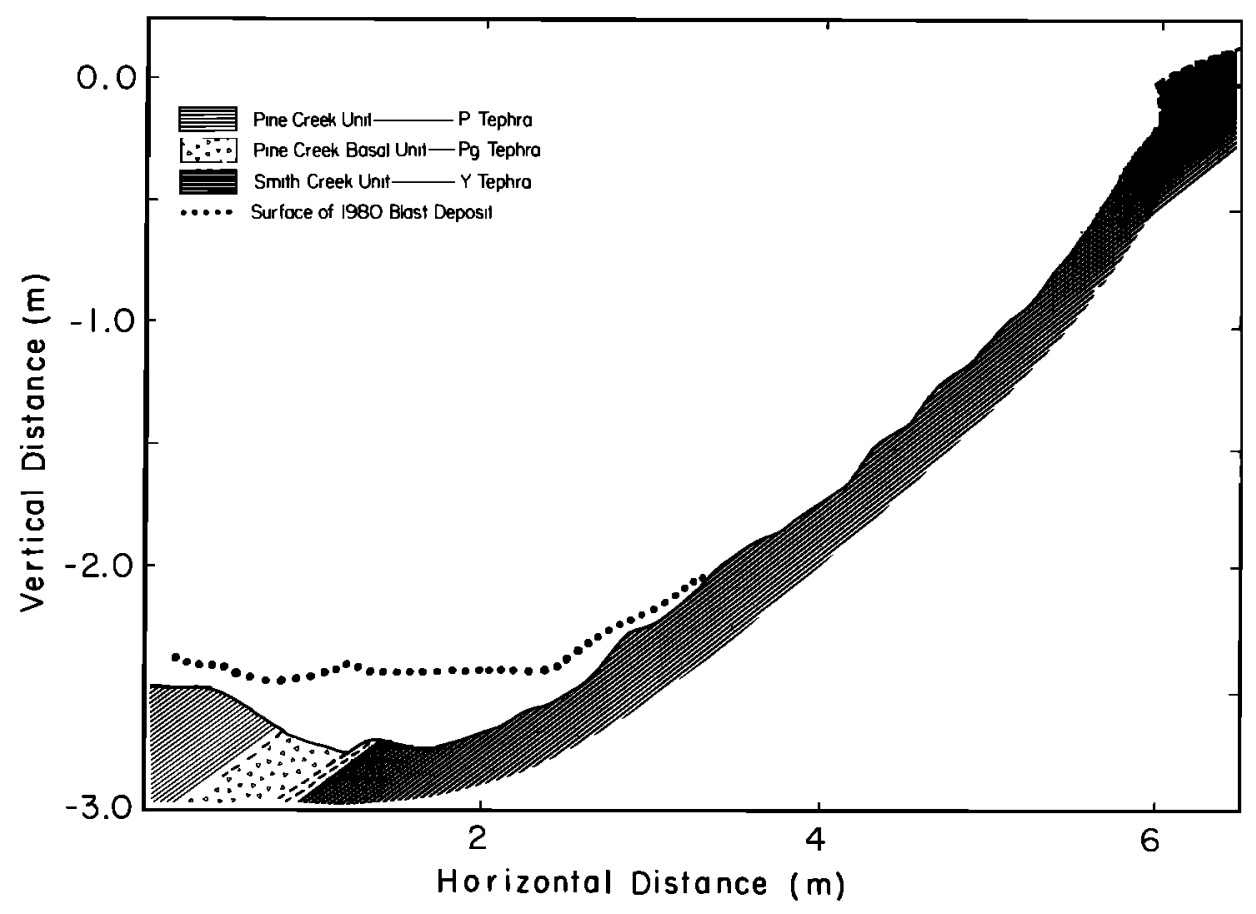

Fig. $14 c$

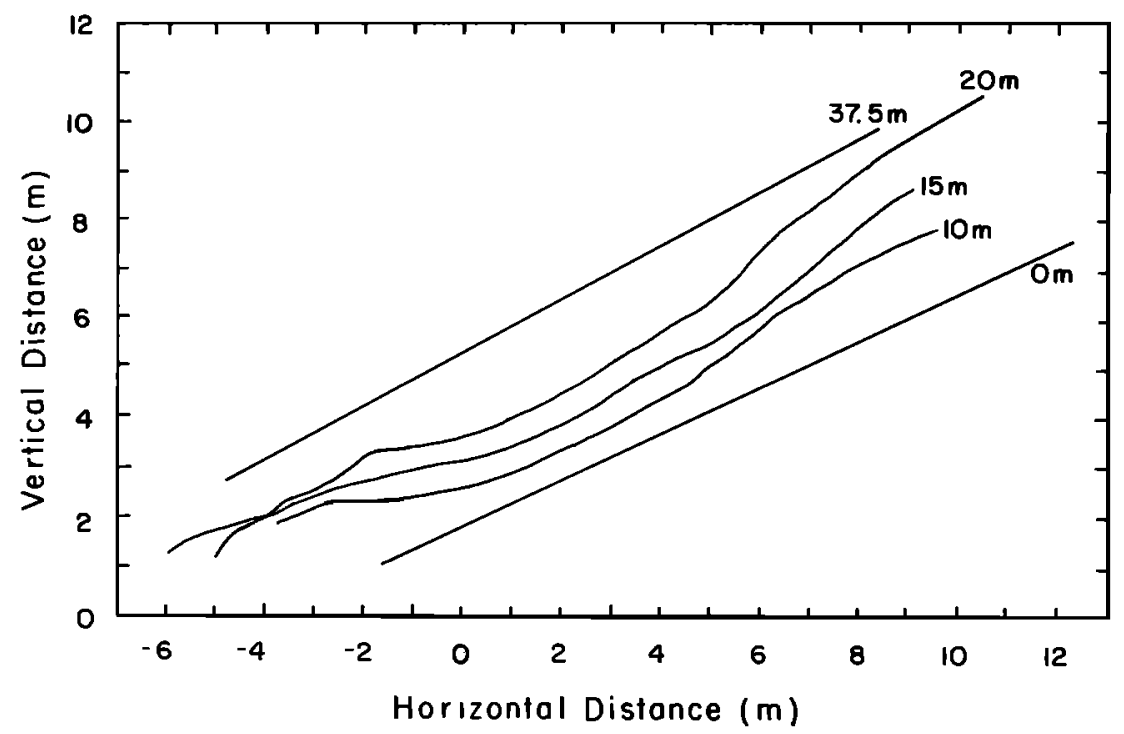

Fig. $14 d$

Fig. 14. (continued)

Johnston Ridge location, where the topography becomes strongly convex about $120 \mathrm{~m}$ downstream of reattachment (Figure 3).

Note that there is no information from the wavelength of the vortices about the free-stream velocity in turbulent flow. For this we turn to the central ridge and some estimates of the conditions under which cross flow produces vortices.

\subsection{Mach Number and Flow Velocity at the Central Ridge}

In Figure 11 more than 15 furrows can be distinguished near or crossing the crest of the central ridge. Relative to the ridge crest they lie at angles ranging from $11^{\circ}$ to $33^{\circ}$, with an average value of $21^{\circ}$. The furrows on the west (windward) side of the ridge lie at somewhat smaller angles than on the east (leeward) side, in a pattern conforming to that of the spiral grooves of Figure 15b. Furthermore, as stated in section 2, the average depths increase from $11 \mathrm{~cm}$ on the west to $16 \mathrm{~cm}$ on the east, i.e., in the downstream direction for spiraling furrows. The similarity between the furrows and the upwash patterns of McDevitt and Mellenthin [1969] suggests that the geometry of the furrows can be used to obtain quantitative estimates of flow properties. As described in section 3, we represent the central ridge as a cone of $16^{\circ}$ half angle at $15^{\circ}$ angle of attack 


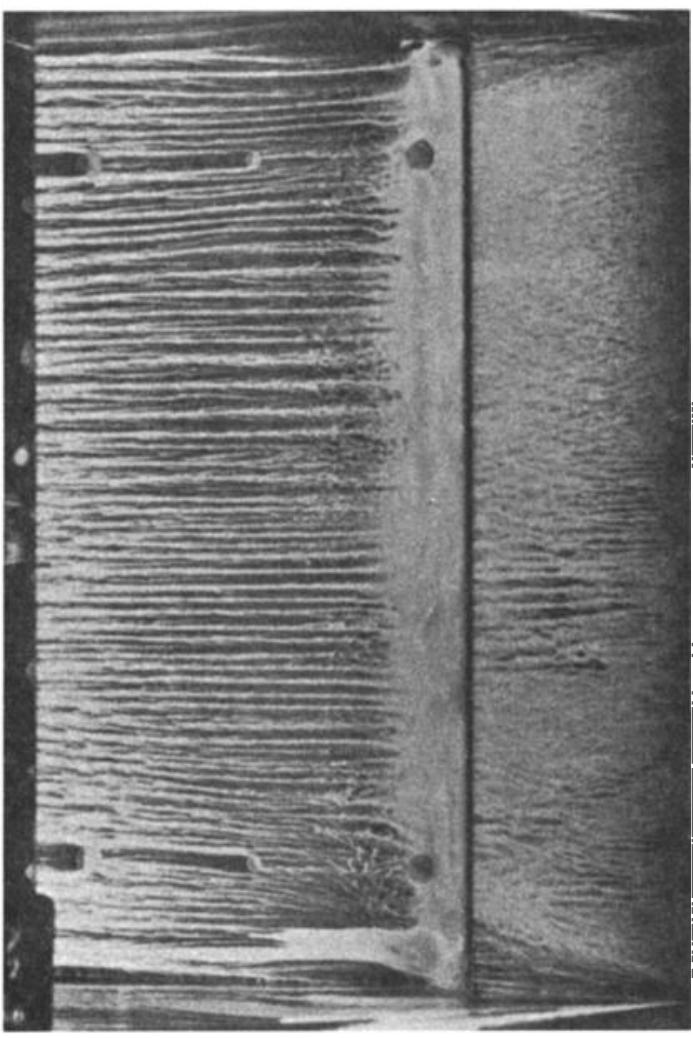

Fig. $15 a$

Fig. 15. (a) Plan view of the flow of air from right to left at Mach 3.0 over a backward facing step mounted on a plate whose leading edge is at the right of the picture [from Hopkins et al., 1960]. Fluorescent oil initially painted smoothly on the plate forms streaks, indicating the presence of vortices. The vertical dark line right of center is the step from which the flow separates, and the smooth light band to the left of the step shows the extent of the region of separated flow. The vortices originate at the line of reattachment at the left edge of the separated flow region. This behavior typifies that at many locations at Mount St. Helens downstream of topographically sheltered regions, best exemplified at locality $\mathrm{HI}$ on Harry's Ridge. Compare with Figures $4 a$ and $4 b$. (b) Photograph of the ablation pattern on an $\mathrm{NH}_{4} \mathrm{Cl}$ cone of $10^{\circ}$ half-angle tested at $10^{\circ}$ angle of attack in air with stagnation temperature $1100 \mathrm{~K}$ and stagnation pressure 102 bars flowing at Mach number 7.4 [from McDevitt and Mellenthin, 1969]. The body was exposed to the hypersonic stream for about $60 \mathrm{~s}$. The figure is oriented to simulate (top) the map view of the central ridge locality with north to the left, and (bottom) the elevation view from the west. The bottom edge of the figure corresponds to the surface of Spirit Lake. The arrow shows the direction of air flow in the wind tunnel test. In the analogy with Mount St. Helens, an observer sitting at the cross on the cone would see the view of Figure $6 a$.

$\alpha$. We assume that the furrow-forming vortices are so large that their orientation reflects that of the flow outside the boundary layer, so we can use theories which neglect the effects of viscosity (i.e., the boundary layer) to relate the flow inclination to other flow properties. In particular, we use the inferred angle between the flow direction at the crest and the line of the crest, known as the "upwash angle" $\varepsilon$, to estimate the Mach number. Approximate theory for slender bodies predicts that $\varepsilon$ is twice $\alpha$ for all free stream Mach numbers $M$, but exact inviscid characteristics theory shows that for cones as slender as $5^{\circ}$ (half angle), $\varepsilon$ can be considerably smaller than $2 \alpha$, depending on $M$. On the other hand, according to theories which account for viscous effects, the upwash angle of fluid deep in the boundary layer adjacent to the body surface can be an order of magnitude greater than $\alpha$. However, in subsonic experiments with yawed cylinders [Poll, 1985], the inclination of oil streaks visualizing cross-flow-induced vortices is typically only about $5^{\circ}$ greater than the stream direction. This, together with the fact that the observed mean inclination of the furrows on the central ridge is substantially less than twice the angle of attack, indicates that the orientation at which furrows will form is not sensitive to motions within the boundary layer very close to the ground.

Using Figure 4 of McDevitt and Mellenthin [1969] for air (ratio of specific heats $\gamma=1.4$ ) with the observed value $\varepsilon \approx 1.4 \alpha$, we find $M \approx 2.5$. Neglecting the effect of the boundary layer on furrow orientation, as we have done here, yields a lower bound for $M$. For example, if we were to utilize the observation of Poll [1985] mentioned above, and take $\varepsilon$ just outside the boundary layer to be, say, $5^{\circ}$ less than the furrow inclination, so $\varepsilon \approx 1.1 \alpha$, then we would get $M \approx 3.7$. On the other hand, though the data of McDevitt and Mellenthin suggest that the dependence on $\gamma$ is quite weak for these conditions, for a smaller $\gamma$ appropriate for the multiphase blast medium at Mount St. Helens, the resulting value of $M$ would be slightly less. Kieffer [1981, Figure 224] calculated a value $M=3.3$ at the location of the central ridge. With a sound speed of $94 \mathrm{~m} / \mathrm{s}$ [Kieffer, 1981], the result $M_{s}=2.5$ implies that the blast flow velocity at the central ridge was of the order of $235 \mathrm{~m} / \mathrm{s}$. Although this is approximately twice the velocity measured for the advance of the front of the blast, it is known that flow expansion from the jet source can accelerate the fluid in an underexpanded jet to velocities substantially larger than that of the flow head [Kieffer and Sturtevant, 1984].

\subsection{Reynolds Number of the Flow}

To evaluate the Reynolds number based on the boundary layer thickness $\delta$,

$$
R e_{\delta}=\frac{\rho U \delta}{\mu_{v}}
$$

we use the following estimates: $U=235 \mathrm{~m} / \mathrm{s}$ (based on the results of section 4.2) and $\delta=14 \mathrm{~m}$ (see beginning of section 4). We adopt a preferred value $\rho=10 \mathrm{~kg} / \mathrm{m}^{3}$ for the flow density. With these values, $R e_{\delta} \approx 1.9 \times 10^{9}$. This choice of $\rho$, together with $\rho_{v}$ given in section 3 , implies that the mass loading, $\kappa \equiv \rho_{s} / \rho_{v}$, is approximately 23 , nearly equal to that assumed by Kieffer [1981].

It is interesting that the value we have adopted for the thickness of the boundary layer implies a global flow scale or "running length" that is consistent with the scale of the Mount St. Helens blast. Results from two different equations for the distance from the source in which a boundary layer of thickness $\delta$ will develop actually bracket the observed distances to furrow locations. The first equation is derived from the "law of the wake" and the "momentum-integral relation" for turbulent boundary layers in pure phases, with numerical factors evaluated from experiments on smooth flat plates [e.g., White, 1974, equations (6-116) and (6-117)];

$$
R e_{x} \approx 8.4 R e_{\delta}^{7 / 6}
$$

where $R e_{x}$ is the Reynolds number based on $x, R e_{x}=\rho U x / \mu_{v}$ With the above numerical values, (5) gives $R e_{x} \approx 5.7 \times 10^{11}$ and $x \approx 4.2 \mathrm{~km}$. The second equation is the Blasius equation, 


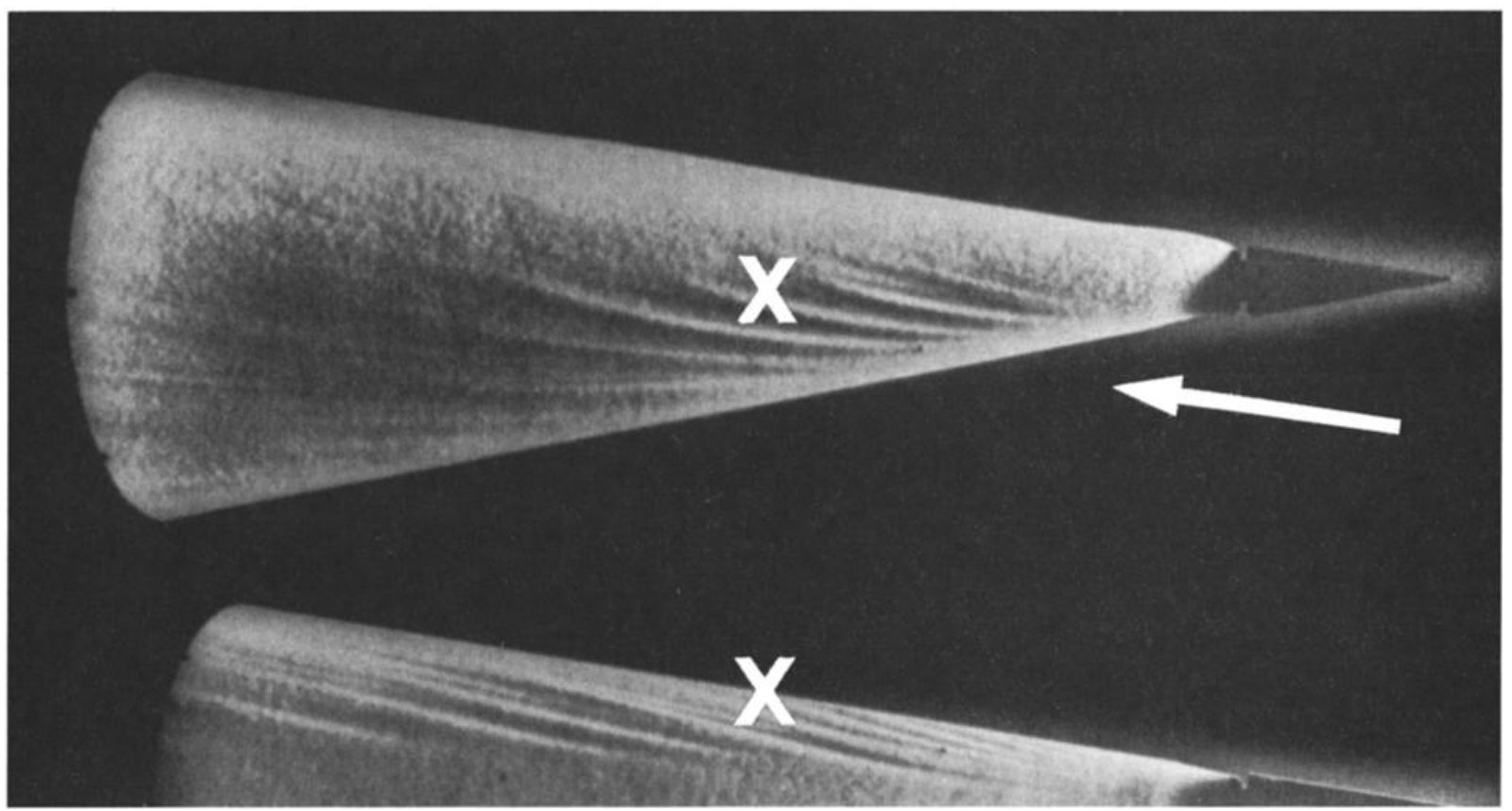

Fig. $15 b$

derived from experiments on turbulent pipe flow [Schlichting, 1955, equation (21.8)]. In a form useful for present purposes it is

$$
x=3.5 \delta \operatorname{Re}_{\delta}^{1 / 4}
$$

In this case the same numerical values as used in (5) yield $x=10.3 \mathrm{~km}$. These two results are close to the minimum and maximum distances at which furrows have been observed at Mount St. Helens. According to White [1974], at the high Reynolds numbers of interest here, (5) should be more accurate than (6).

\subsection{Erosion Model}

We next use an empirical model for the rate of erosion under a turbulent boundary layer in high-speed flow. The depth $\Delta$ of material removed during the erosive phase of the lateral blast is

$$
\Delta=\frac{\dot{m} t}{d_{s}}
$$

where $\dot{m}$ is the time-average vertical mass flux of eroded soil per unit surface area. For this calculation we treat only the measurable erosion defined by the crests and troughs of the furrows, and we do not account for possible additional erosion above the present furrows mentioned at the end of section 2 . In laboratory and field experiments with lightly loaded flows [Bagnold, 1942; Hartenbaum, 1971] it has been found that the mass removal rate of sand by wind shear from a horizontal bed is proportional to the product of the flow density and the so-called friction velocity,

$$
u_{\tau}=(\tau / \rho)^{1 / 2}
$$

where $\tau$ is the shear stress exerted by the flow on the ground:

$$
\dot{m}=K \rho u_{\tau}
$$

$K$, an empirically determined constant, is the "lofting ef- ficiency." The data of Hartenbaum [1971] indicate that, in lightly loaded flows with uniform vortex-free boundary layers at velocities up to $115 \mathrm{~m} / \mathrm{s}$, the lofting efficiency, which for this case we designate as $K_{V F}$, is approximately 0.05 . We derive this value from the information provided by Hartenbaum [1971], by taking the particle density in his flow to be $10^{8}$ $\mathrm{m}^{-3}$ and the bulk density of his particulate (Ottawa sand) to be $2.5 \times 10^{3} \mathrm{~kg} / \mathrm{m}^{3}$, yielding a local flow density $\rho=2.8$ $\mathrm{kg} / \mathrm{m}^{3}$. With a slightly different interpretation, Hartenbaum reports $K \approx 0.1$.

To estimate the friction velocity, we invoke the logarithmic friction law obtained from turbulent boundary layer theory [e.g., Tennekes and Lumley, 1972, p. 186; White, 1974, p. 483],

$$
\frac{U}{u_{\mathrm{r}}}=\frac{1}{\kappa_{\mathrm{K}}} \ln \frac{\rho u_{\mathrm{r}} \delta}{\mu_{v}}+A
$$

where $\kappa_{K}=0.4$ is Karman's constant and $A \approx 8$ for flat plates. Thus, $u_{\tau} / U$ is a weak function of $\mu, \rho$, and $\delta$; taking the preferred values cited above, $u_{\tau} / U \approx 0.019$. From (8) and (9),

$$
\Delta=K U t \frac{\rho}{d_{s}} \frac{u_{\tau}}{U}
$$

and we see from (10) and (11) that the material removed depends linearly on $K$ and $t$, nearly linearly on $U$ and $\rho$, and very weakly on $\delta$ and $\mu$. Note that the flow variables upon which $\Delta$ depends most strongly are just the ones that are particularly poorly constrained in volcanic blasts. Furthermore, $K$ is not known for naturally occurring flows and might vary from the small values observed in the laboratory to, perhaps, greater than 1 . Using the ranges of variables cited earlier in this section, the possible range of the product $K t U \rho$ is from $5 \times 10^{-1}$ to $2.5 \times 10^{7} \mathrm{~kg} / \mathrm{m}^{2}$ !

To exhibit the possible variations, we have plotted $\Delta$ versus $K U t \rho / d_{s}$ in Figure 16. In the figure we have neglected small variations of $u_{\tau} / U$, using the preferred value given above. To the extent that the value adopted is representative, the re- 


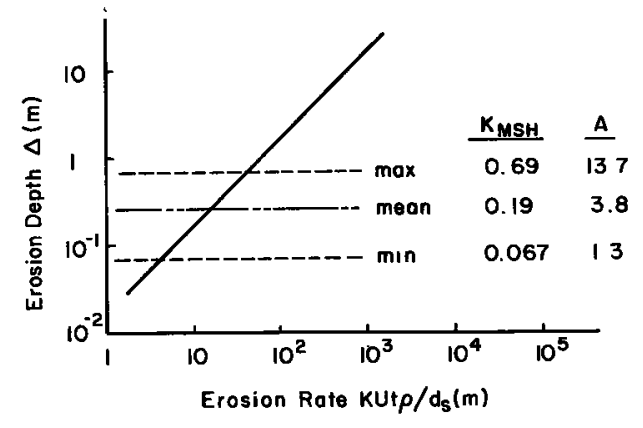

Fig. 16. Erosion depth as a function of erosion rate parameter $(K$, lofting efficiency; $U$, free stream velocity; $t$, flow duration; $\rho$, flow density; and $d_{s}$, bulk density of material being eroded). Solid line shows the relationship given by (11) with $u_{\mathrm{r}} / U=0.019$. Dashed lines show limits of observed erosion $\Delta$ in furrows at Mount St. Helens (Table 2). Chained line shows mean value of depths reported in Table

sulting straight-line relation in Figure 16 should yield a valid estimate of erosion depth for volcanic blasts which do not differ in scale by orders of magnitude from that at Mount St. Helens. Also shown in Figure 16 is the range of observed values and the average of the mean depths recorded in Table 2. The intersections of these lines with the solid line can be used, with preferred values already quoted and a preferred flow time $t=30 \mathrm{~s}$, to give a range of lofting efficiency $K$ appropriate for the Mount St. Helens blast. The results are given in the figure, in the column headed $K_{M S H}$. The ratio of $K_{M S H}$ to the efficiency observed in vortex-free laboratory flows, $A=K_{M S H} / K_{V F}$, is also given in the figure. The results show that the longitudinal furrow-producing vortices at Mount St. Helens enhanced erosion over that observed in uniform vortex-free flow by a factor of from 1.3 to 14 . The average of the observed furrow depths reported in section 2 (which yields $A=3.8$ ) and the preferred values of $d_{s}$ and $t$ imply that the scouring rate at Mount St. Helens was about $0.7 \mathrm{~cm} / \mathrm{s}$ and $\dot{m}$ was of the order of $9 \mathrm{~kg} \mathrm{~m}^{-2} \mathrm{~s}^{-1}$. In one large furrow for which the volume was estimated, J2-15, the erosion rate was of the order of $7 \mathrm{~m}^{3} / \mathrm{s}$, or $9 \mathrm{t} / \mathrm{s}$ ! Since the data set from which the average depth is derived is very limited, these results are presented only to give an idea of the magnitude of the effects. In view of the fact that the observed furrow depths are only a lower bound on the actual erosion caused by the furrowing mechanism, the resulting accuracy should be sufficient for present purposes.

\section{Conclusions}

Furrowing during the erosional phase of the blast at Mount St. Helens has been attributed to large high-energy longitudinal vortices in the boundary layer of the blast flow. The analysis presented here shows which variables are most important in determining the erosion by the vortices; many of them are the variables most poorly constrained by observations. The orientation of the furrows records the flow direction, while their size gives the boundary layer thickness, $\delta \approx 14 \mathrm{~m}$. From the orientation of the central ridge relative to the mountain and of the furrows on that ridge it is possible to evaluate the flow Mach number there; $M \approx 2.5$. With an estimate of the local sound speed, the velocity is estimated to be about 235 $\mathrm{m} / \mathrm{s}$. This information, together with an estimate of the viscosity, is sufficient to determine the flow Reynolds number based on boundary layer thickness; $R e_{\delta} \approx 1.9 \times 10^{9}$. The inferred thickness of the boundary layer at locations ranging from 3.5 to $9 \mathrm{~km}$ from the mountain is consistent with the thickness predicted by two classical formulas derived from laboratory experiments on the flow of single-phase fluids over smooth plates and in pipes. In spite of the uncertainty of many of the variables, the erosion under the vortices appears to have been up to 1 order of magnitude larger than would have been predicted for a vortex-free boundary layer at the same flow conditions. Analysis of measurements of furrow depth, combined with these estimates of flow velocity, density, and duration, yields the erosional efficiency of the flow, $K \approx 0.2$. Note that if values of $\rho, U$, or $t$ larger than those assumed or inferred here were adopted, so that the product $t U \rho$ were much larger, then the small erosional efficiency implied by Figure 16 would be inconsistent with the enhanced erosion that actually occurred in the furrows (averaging about $20 \mathrm{~cm}$ ), while if $t U \rho$ were much smaller, the implied efficiency would seem to be unreasonably large (i.e., greater than 1). On the other hand, a simultaneous decrease of, say, $\rho$ and increase of $U$, such that $t U \rho$ remains essentially unchanged, cannot be distinguished in this analysis.

The furrows that occur from 3.5 to $9 \mathrm{~km}$ from the origin of the blast at Mount St. Helens are always associated with ridges and are found at locations where the flow orientation was subhorizontal. Furrows of similar shape, size, and number have been observed as radial channels on the uniformly sloping flanks of conical volcanoes (see section 1). A spectacular example, showing many erosional furrows of nearly sinusoidal cross section and regular spacing, was given by Richards [1959, Plate 21] (Figure 17). Their shape is similar to the sketch of longitudinal dunes at Cerro Colorado, Mexico, by Wohletz [1977, Figure 44], who hypothesized that at that locale the troughs were eroded and the crests were deposited by longitudinal vortices. However, furrows have not been recognized as far from the source or in as complex terrain as at Mount St. Helens. This could be due to the fact that high velocities and densities sufficient to cause major erosion at

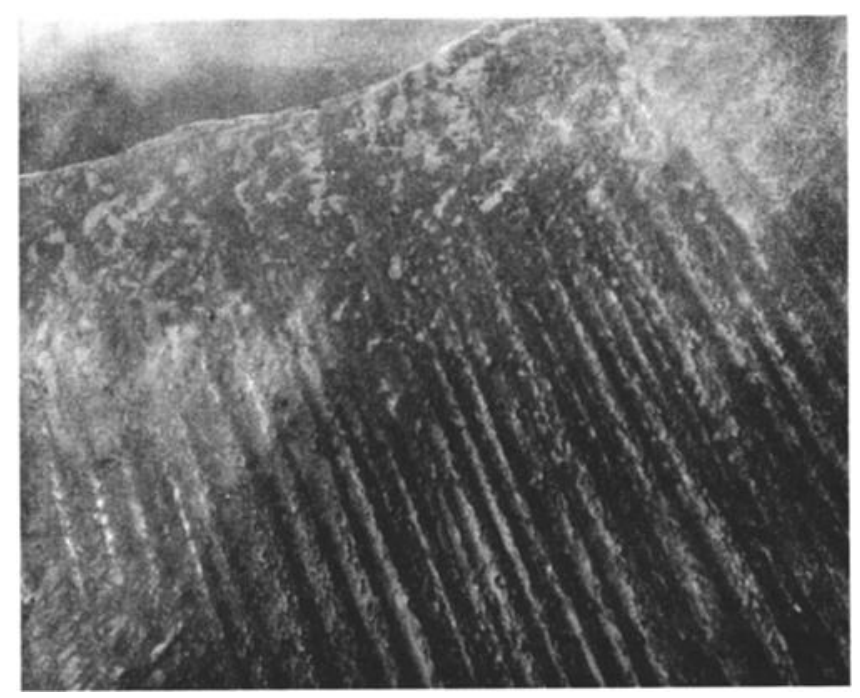

Fig. 17. Aerial photo of furrows near the rim of Bárcena Volcano. From Plate 21 of Richards [1959], captioned, "furrow detail on the east flank of Bárcena. Upper ends of the furrows." 
large distance are only found in high-energy lateral blasts, and that such laterally directed flows rarely occur, or that the debris avalanches which accompany lateral blasts usually leave overlying and/or obscuring deposits, or that furrows in steep and complex terrain are particularly hard to recognize after they become eroded. Nevertheless, radial channels on volcanic cones may be related to the reattachment mechanisms discussed in this paper if the channels were formed, for example, during collapse of a Plinian column, when flowing material would fall back onto the slopes. In such a case, the curvature of the flow required for the formation or intensification of streamwise vortices would be provided by the change of flow direction from subvertically downward to parallel to the slope. Intensification or lack of intensification of vortices under these conditions may have significant implication not only for erosion and dune formation, but also for the distance at which some characteristic near-vent deposits (e.g., lag breccias) are produced, because the enhanced erosive ability implied by the presence of large-scale vortices may influence the distance at which the transition from erosional to depositional conditions can occur. In view of the fact that the region around many volcanoes is heavily populated at $10-\mathrm{km}$ distances, the factor of 4 enhancement of erosion by longitudinal vortices inferred in this work may have significant implications for the engineering design of protective structures in zones where furrowing may occur. It is hoped that the work presented here will lead not only to further examination of the possibility of furrows at both proximal and distal locations at other volcanoes, but also to further investigations of their function and of the implications of coupling between topography and flow.

Acknowledgments. We thank Harry Glicken, Richard Hoblitt, and Don Mullineaux for useful conversations, Hans Hornung and Michael Sheridan for penetrating reviews of the manuscript, and Ichiro Sugioka for assistance in surveying, data reduction, and preparation of the manuscript. This work was supported jointly by the National Science Foundation under grant EAR-8512724 and the U.S. Geological Survey. Approved by Director, U.S. Geological Survey, November $5,1987$.

\section{REFERENCES}

Bagnold, R. A., The Physics of Blown Sands and Desert Dunes, Morrow, New York, 1942.

Bernal, L. P., and A. Roshko, Streamwise vortex structure in plane mixing layers, J. Fluid Mech., 170, 499, 1986

Brown, G. L., and A. Roshko, The effect of density difference on the turbulent mixing layer, Turbulent Shear Flows, AGARD-CP-93, p. 23-1, Advis. Group for Aeron. Res. and Dev., Brussels, 1971.

Brown, G. L., and A. Roshko, Density effects and large structure in turbulent mixing layers, J. Fluid Mech., 64, 775, 1974.

Canning, T. N., M. E. Wilkens, and M. E. Tauber, Ablation patterns on cones having laminar and turbulent flows, $A I A A J ., 6,174$ 1968.

Crandell, D. R., and D. R. Mullineaux, Pine Creek volcanic assemblage at Mount St. Helens, Washington, U.S. Geol. Surv. Bull., I383- $A, 1973$

Darwin, C., Geological Observations on the Volcanic Islands and Parts of South America Visited During the Voyage of H.M.S. 'Beagle', 3rd ed., 648 pp., D. Appleton, New York, 1896. (AMS Press, New York, 1972).

Fisher, R. V., Erosion by volcanic base-surge density currents: Ushaped channels, Geol. Soc. Am. Bull., 88, 1287, 1977.

Fisher, R. V., and H.-U. Schminke, Pyroclastic Rocks, SpringerVerlag, New York, 1984.

Fisher, R. V., H. X. Glicken, and R. P. Hoblitt, May 18, 1980, Mount St. Helens deposits in South Coldwater Creek, Washington, J. Geophys. Res., 92, 10,267-10,283, 1987.
Ginoux, J., Experimental evidence of three-dimensional perturbations in the reattachment of a two-dimensional laminar boundary layer at $M=2.05$, TCEA TN 1, Training Cent. for Exp. Aerodyn., Rhode-Saint-Geriese, Belgium, 1958.

Görtler, $H_{\text {., }}$ On the three-dimensional instability of laminar boundary layers on concave walls (translation), NACA Tech. Memo., TM $1375,1954$.

Gregory, N., J. T. Stuart, and W. S. Walker, On the stability of three-dimensional boundary layers with application to the flow due to a rotating disk, Philos. Trans. R. Soc. London, Ser. A, 248, 155, 1955.

Hartenbaum, B., Lofting of particulates by a high speed wind, Rep. DNA-2737, Def. Nucl. Agency, Washington, D. C., 1971.

Hopkins, E. J., S. J. Keating, and A. Bandettini, Photographic evidence of streamwise arrays of vortices in boundary-layer flow, NASA Tech. Note, TN D-328, 1960.

Kieffer, S. W., Fluid dynamics of the May 18 blast at Mount St. Helens, U.S. Geol. Surv. Prof. Pap., 1250, 379, 1981.

Kieffer, S. W., and B. Sturtevant, Laboratory studies of volcanic jets, J. Geophys. Res., 89, 8253, 1984.

Konrad, J. H., An experimental investigation of mixing in twodimensional turbulent shear flows with applications to diffusionlimited chemical reactions, Proj. Squid Tech. Rep. CIT-8-PU, Purdue Univ., West Lafayette, Indiana, 1976.

Lipman, P. W., and D. R. Mullineaux (Eds.), The 1980 eruptions of Mount St. Helens, Washington, U.S. Geol. Surv. Prof. Pap., 1250, 844 pp., 1981.

Losacco, U., and G. C. Parea, Saggio di un atlante di strutture sedimentarie e postsedimentarie osservate nelle piroclastiti del Lazio, Atti Soc. Nat. Mat. Modena, 94, 330 pp., 1969.

Marble, F. E., Dynamics of dusty gases, Annu. Rev. Fluid Mech., 2, 397, 1970.

Mattson, P. H., and W. Alvarez, Base surge deposits in Pleistocene volcanic ash near Rome, Bull. Volcanol., 37, 553, 1973.

McDevitt, J. B., and J. A. Mellenthin, Upwash patterns on ablating and nonablating cones at hypersonic speeds, NASA Tech. Note, TN D-5346, 1969.

Moore, J. G., Base surge in recent volcanic eruptions, Bull. Volcanol., $30,337,1967$

Moore, J. G., and T. W. Sisson, Deposits and effects of the May 18 pyroclastic surve, U.S. Geol. Surv. Prof. Pap., 1250, 421, 1981.

Mullineaux, D. R., J. H. Hyde, and M. Rubin, Widespread late glacial and postglacial tephra deposits from Mount St. Helens Volcano, Washington, J. Res. U.S. Geal. Surv., 3, 329, 1975.

Persen, L. N., Surface patterns of ablating bodies studied by means of water experiment simulation, Z. Flugwiss., 19, 360, 1971.

Poll, D. I. A., Some observations of the transition process on the windward face of a long yawed cylinder, J. Fluid Mech., 150, 329, 1985.

Richards, A. F., Geology of the Islas Revillagigedo, Mexico, 1, Birth and development of Volcan Barcena, Isla San Benedicto, Bull. Vol canol., 22, 73, 1959.

Roshko, A., Structure of turbulent shear flows, AIAA J., 14, 1349, 1976.

Rowley, P. D., M. A. Kuntz, and N. S. MacLeod, Pyroclastic-flow deposits, U.S. Geol. Surv. Prof. Pap., 1250, 489, 1981.

Schlichting, H., Zur Entstehung der Turbulenz bei der Plattenströmung, Nachr. Ges. Wiss. Göttingen Math Phys. Kl., 160198, 1933. (Amplitude distribution and energy balance of small disturbances in plate flow, NACA Tech. Memo., TM 1265, Engl. Transl., 1950.)

Schlichting, H., Boundary Layer Theory, McGraw-Hill, New York, 1955.

Sigurdson, L. W., The structure and control of a turbulent reattaching flow, Ph.D. thesis, Calif. Inst. of Technol., Pasadena, 1986.

Simons, D. B., E. V. Richardson, and C. F. Nordin, Jr., Sedimentary structures generated by flow in alluvial channels, Primary Sedimentary Structures and Their Hydrodynamic Interpretation, Spec. Publ. Soc. Econ. Paleontol. Mineral., 12, 34, 1965.

Stuart, J. T., Hydrodynamic stability, in Laminar Boundary Layers, edited by L. Rosenhead, 492 pp., Oxford University Press, New York, 1963.

Tani, I., Production of longitudinal vortices in the boundary layer along a concave wall, J. Geophys. Res., 67, 3075, 1962.

Taylor, G. I., Stability of viscous liquid contained between two rotating cylinders, Philos. Trans. R. Soc. London, 223, 289, 1923. 
Tennekes, H., and J. L. Lumley, A First Course in Turbulence, MIT Press, Cambridge, Mass., 1972.

Tobak, M., Hypothesis for the origin of cross-hatching, AIAA Pap. 69-11, Am. Inst. of Aeron. and Astron., 1969.

Tollmien, W., Uber die Entstehung der Turbulenz, Nachr. Ges. Wiss. Göttingen, 21-44, 1929. (The production of turbulence, NACA Tech. Memo., TM 609, Engl. Transl., 1931.)

Weedman, S. D., and R. Slingerland, Experimental study of sand streaks formed in turbulent boundary layers, Sedimentology, 32, $133,1985$.

White, F. M., Viscous Fluid Flow, McGraw-Hill, New York, 1974.
Wohletz, K. H., A model of pyroclastic surge, M. Sc. thesis, Ariz. State Univ., Tempe, 1977.

S. W. Kieffer, U.S. Geological Survey, Branch of Igneous and Geothermal Processes, 2255 North Gemini Drive, Flagstaff, AZ 86001.

B. Sturtevant, Graduate Aeronautical Laboratories, California Institute of Technology, Pasadena, CA 91125.

\section{(Received November 11, 1987;} accepted March 10, 1988.) 